THE ASTrophysical Journal SUPPLEMENT SERIES, 98:103-128, 1995 May

(C) 1995. The American Astronomical Society. All rights reserved. Printed in U.S.A.

\title{
ULTRAVIOLET TO NEAR-INFRARED SPECTRAL DISTRIBUTIONS OF STAR-FORMING AND SEYFERT 2 GALAXIES
}

\author{
THAISA STORCHI-BERGMANN ${ }^{1}$ \\ Departamento de Astronomia, IF-UFRGS, CP 15051, CEP 91501-970, Porto Alegre, RS, Brazil \\ ANNE L. KINNEY ${ }^{1,2}$ AND PETER CHALlis ${ }^{1}$ \\ Space Telescope Science Institute, 3700 San Martin Drive, Baltimore, MD 21218 \\ Received 1994 April 29; accepted 1994 July 14
}

\begin{abstract}
Combined UV, optical, and near-IR spectral distributions of a sample of 48 southern star-forming and Seyfert 2 galaxies are presented. The optical and near-IR spectra were obtained using apertures matching those of the UV observations $\left(10^{\prime \prime} \times 20^{\prime \prime}\right)$ and in most cases correspond to the bulk of the gas and stellar population contribution from the whole galaxy. No such data set-taken with matched apertures and spanning such a large spectral range-exists in the literature today. The obtained spectral distributions are particularly useful as a comparison sample for spectroscopic surveys of more distant galaxies. Emission-line fluxes, continuum fluxes, and equivalent widths of absorption lines are measured from the spectra, and average values corresponding to the different activity types-blue compact dwarf, blue compact, starburst, Seyfert, and composite (starburst and Seyfert) - are presented and discussed.
\end{abstract}

Subject headings: galaxies: Seyfert — galaxies: starburst — galaxies: photometry — infrared: galaxies — ultraviolet: galaxies

\section{INTRODUCTION}

In a previous work, Kinney et al. (1993) have produced an atlas of ultraviolet spectra of the central regions of 143 galaxies showing star formation in its nucleus, from observations obtained with the IUE satellite. In order to extend the spectral distributions to the optical and near-IR, ground-based observations have been made using a matched aperture to that of the UV $\left(10^{\prime \prime} \times 20^{\prime \prime}\right)$. In this work we present the data of the southern sample, obtained at the Cerro Tololo Inter-American Observatory (CTIO). With these observations we were able to construct spectral distributions from 1200 to $10,000 \AA$, with an average spectral resolution of $5 \AA$ for 48 star-forming and Seyfert 2 galaxies. Optical spectra of the northern sample were obtained at the Kitt Peak National Observatory and will be presented in McQuade, Calzetti, \& Kinney (1995).

In this work we also provide measurements of emission-line fluxes and equivalent widths of the absorption lines, as well as fluxes at several points of the continuum for the southern sample, which were used to determine the chemical abundance, reddening, star-forming rate, and age of the central regions of the sample galaxies. The study of the dependence of the spectral distributions on the above parameters, for both the southern and northern sample, are presented in two other papers (Storchi-Bergmann, Calzetti, \& Kinney 1994; Calzetti, Kinney, \& Storchi-Bergmann 1994). Galaxies with similar properties are being grouped together in order to construct template spectra (Kinney, Calzetti, \& Storchi-Bergmann 1995). Our goal is to understand the star formation in nearby galaxies and to use the results of our study to predict the properties of star-

\footnotetext{
${ }^{1}$ Visiting Astronomer at the Cerro Tololo Inter-American Observatory, operated by the Association of Universities for Research in Astronomy, Inc., under contract with the National Science Foundation.

${ }^{2}$ Guest investigator with IUE Observatory.
}

forming galaxies at high redshift. The large aperture of our observations usually includes most of the galaxy light, so that our spectral distributions are well suited for comparison with observations of distant galaxies, for which even small apertures include most of the galaxy.

\section{OBSERVATIONS}

Long-slit spectral observations were made at CTIO in three epochs: 1991 October-November, 1992 May, and 1992 November. In order to get an aperture as close as possible to the one from IUE observations, we used a slit width corresponding to $10^{\prime \prime}$ in the sky, and then extracted a window $20^{\prime \prime}$ long. Two telescopes were used in the optical and near-IR observations: the $1 \mathrm{~m}$ telescope with Cassegrain Spectrograph and 2DFRUTTI detector was used to cover the range $\lambda \lambda 3200-$ 6400 with $8 \AA$ resolution, and the $1.5 \mathrm{~m}$ telescope with Cassegrain Spectrograph and a CCD detector to cover the range $\lambda \lambda 6400-10000$ at $5.5 \AA$ resolution. The spectral resolution was chosen to be as close as possible to the one of the UV observations, which are $5 \AA$ for the SWP and $8 \AA$ for the LWP and LWR. A log of the observations is presented in Table 1. The activity types and distances of the galaxies, obtained from Kinney et al. (1993, from the apparent $B$ and absolute $M_{B}$ magnitudes listed on their Table 1), are listed in the second and third columns of the table.

The spectra were reduced using the IRAF software, including flux calibration through observations of standard stars. The near-IR spectra were then corrected for the atmospheric absorption bands of $\mathrm{H}_{2} \mathrm{O}$ and $\mathrm{O}_{2}$ by dividing the galaxies' spectra by the spectra of hot standard stars, after removing the $\mathrm{H} \alpha$ absorption line when present in the stellar spectrum and normalizing the slope of the stellar continuum to 1 . Two examples of the resulting spectral distributions are shown in Figures 1 (a typical blue spectrum) and 2 (a typical red spectrum). 


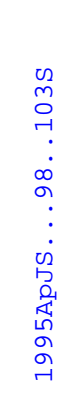

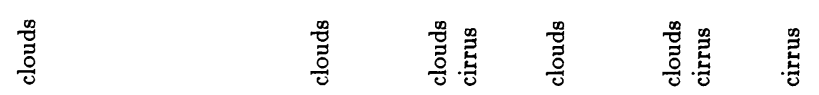

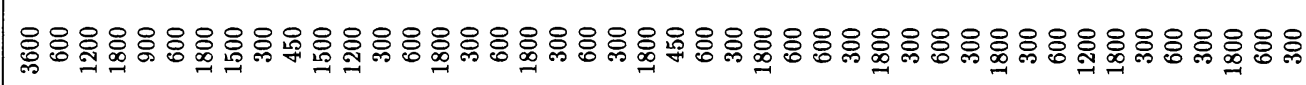

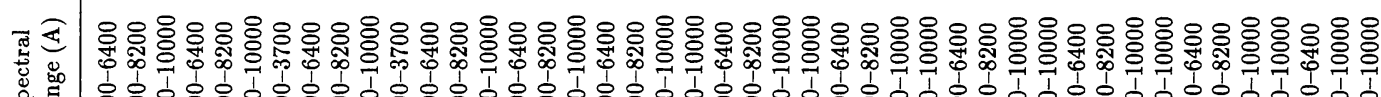

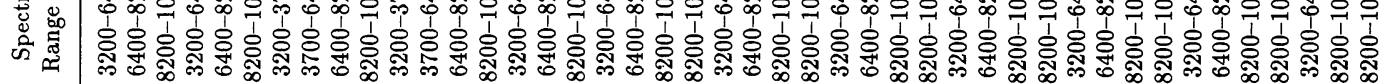

峞

శ్

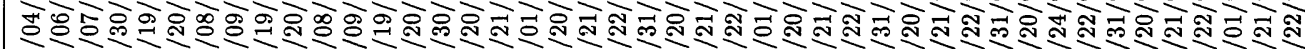

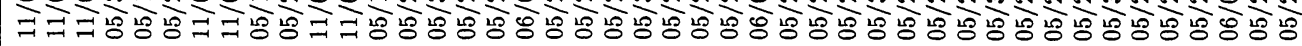

商产旁突

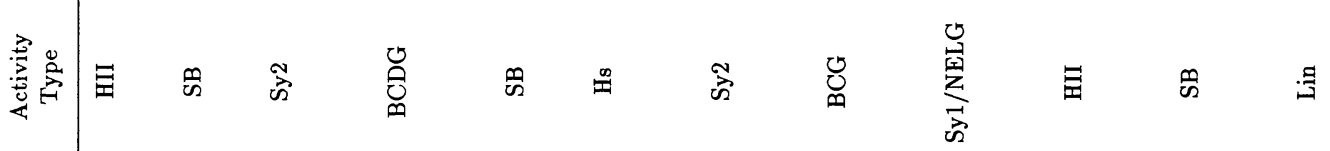

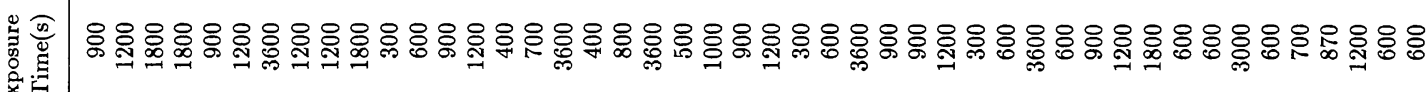
总.

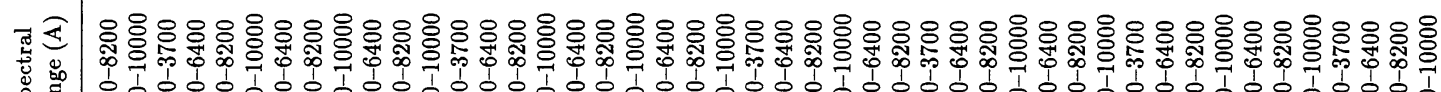

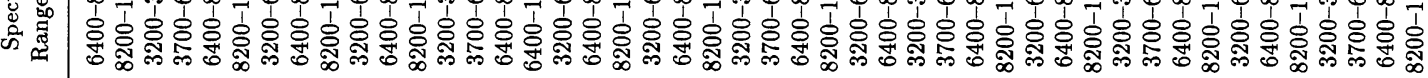

ఎ స్య

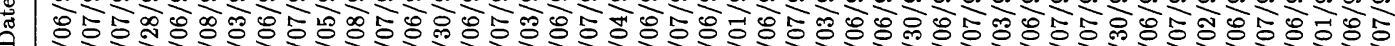

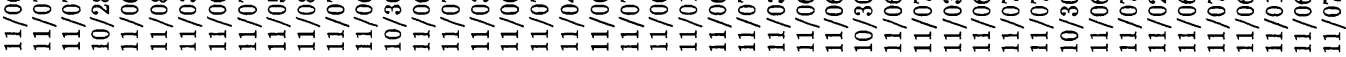

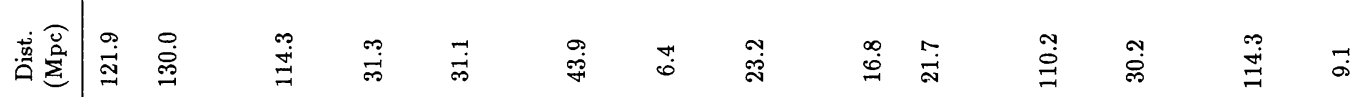

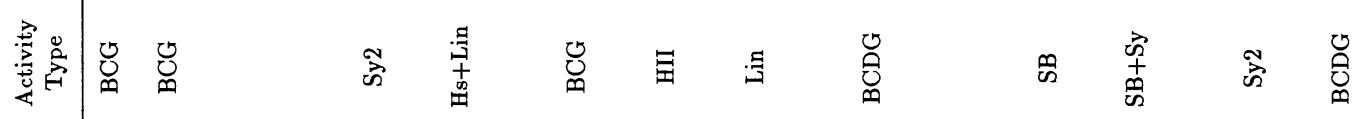

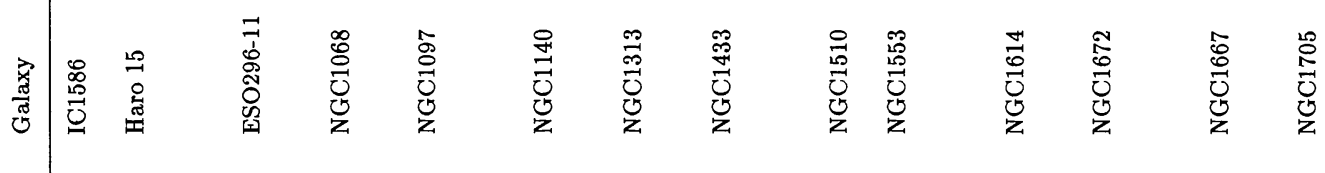




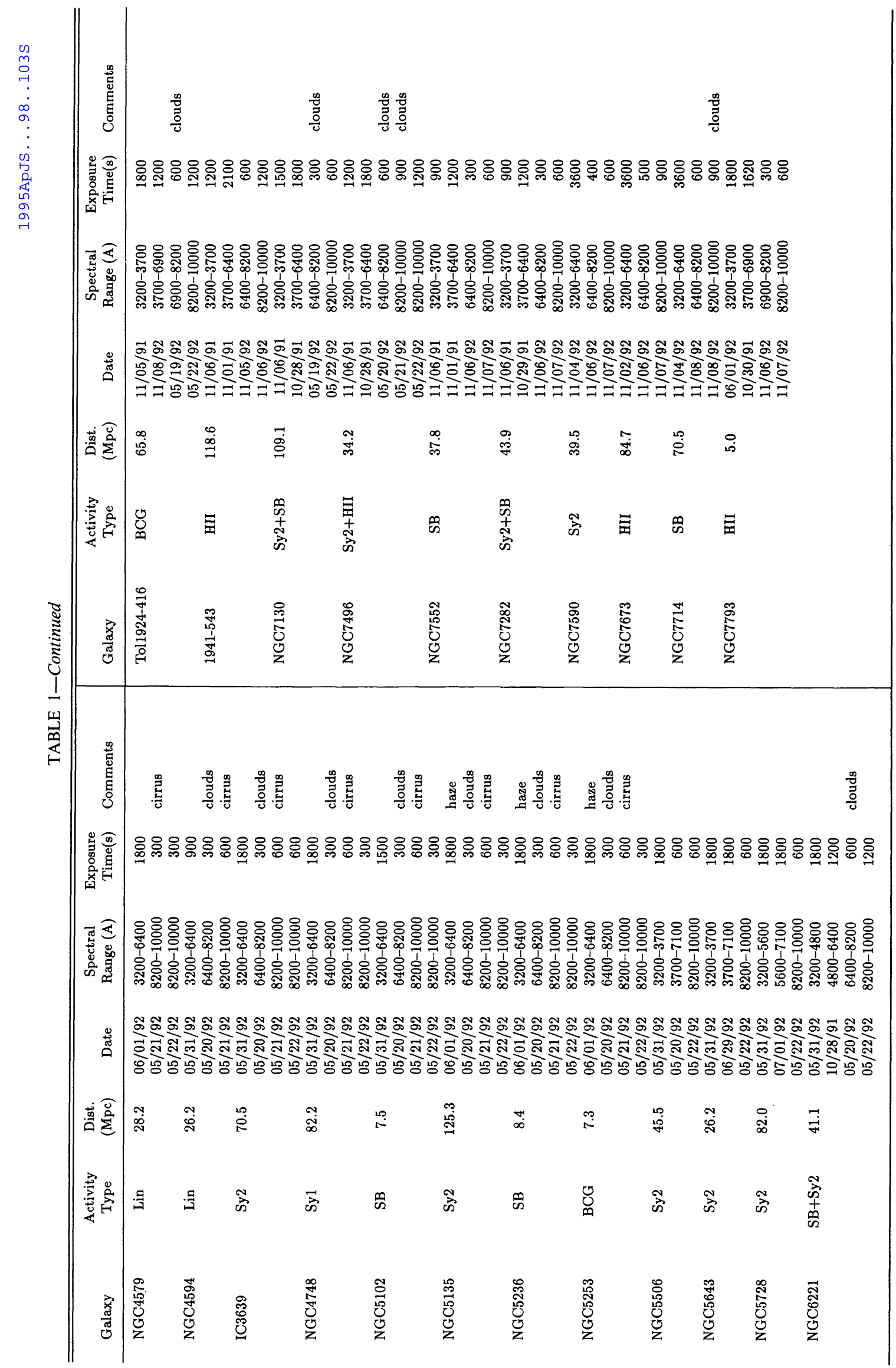




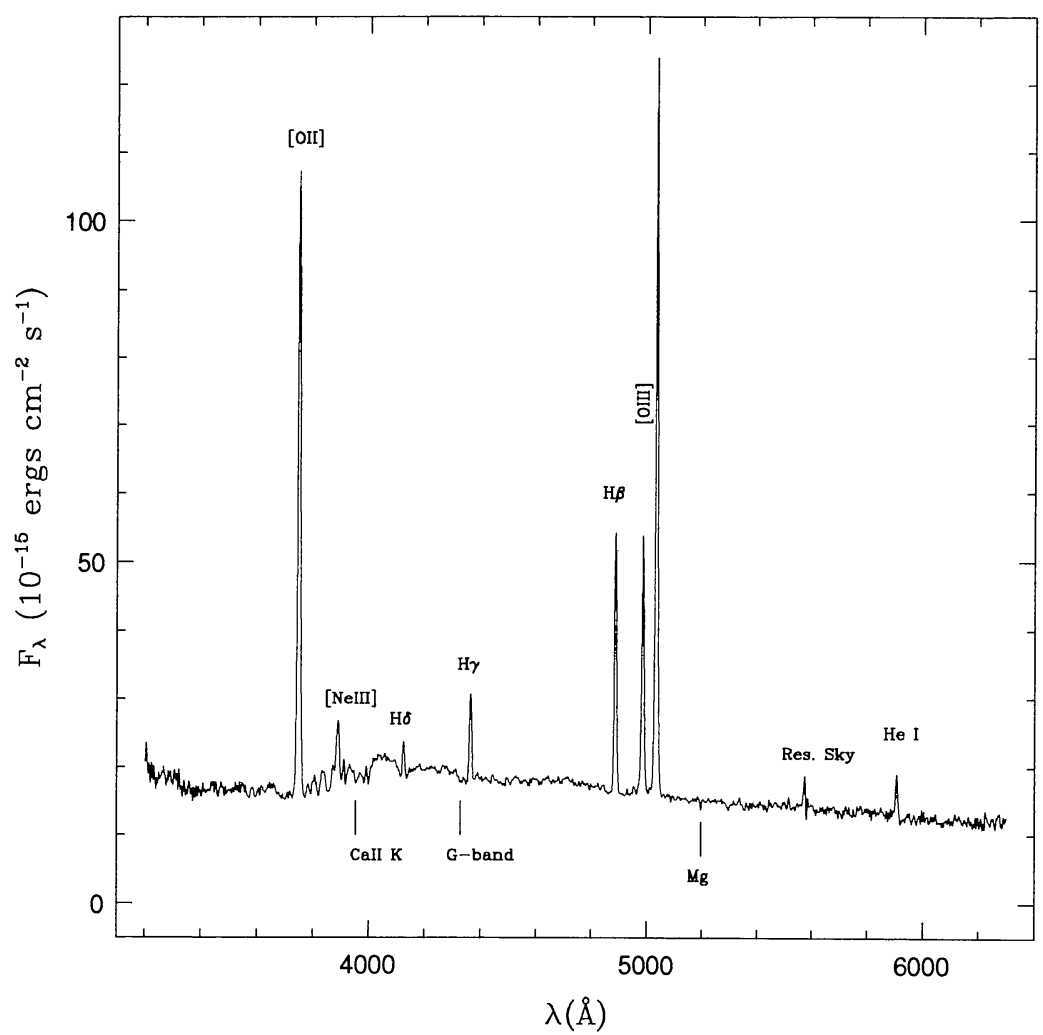

FIG. $1 a$

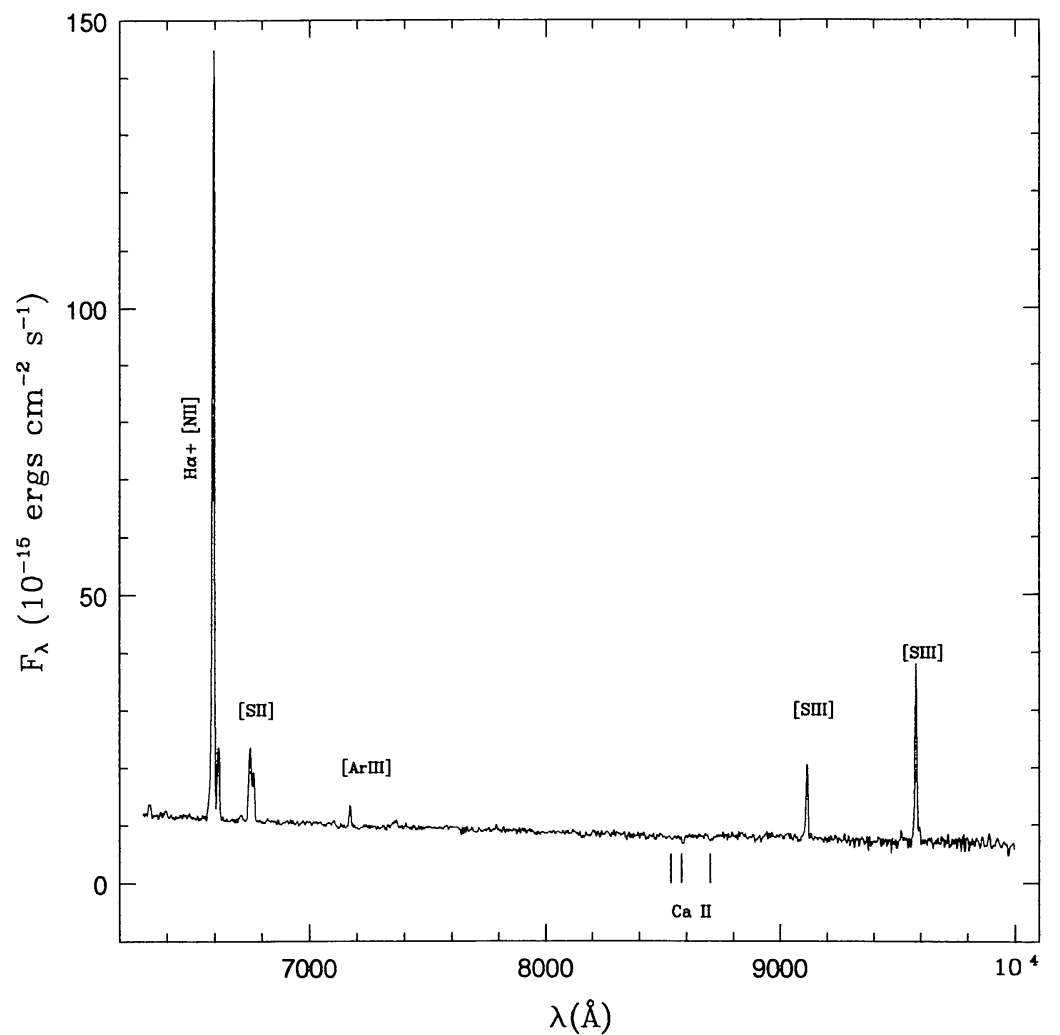

FIG. $1 b$

FIG. 1.-( $a$ ) Optical and $(b)$ near-IR spectrum of the galaxy NGC 1140. The main measured spectral features are identified. 


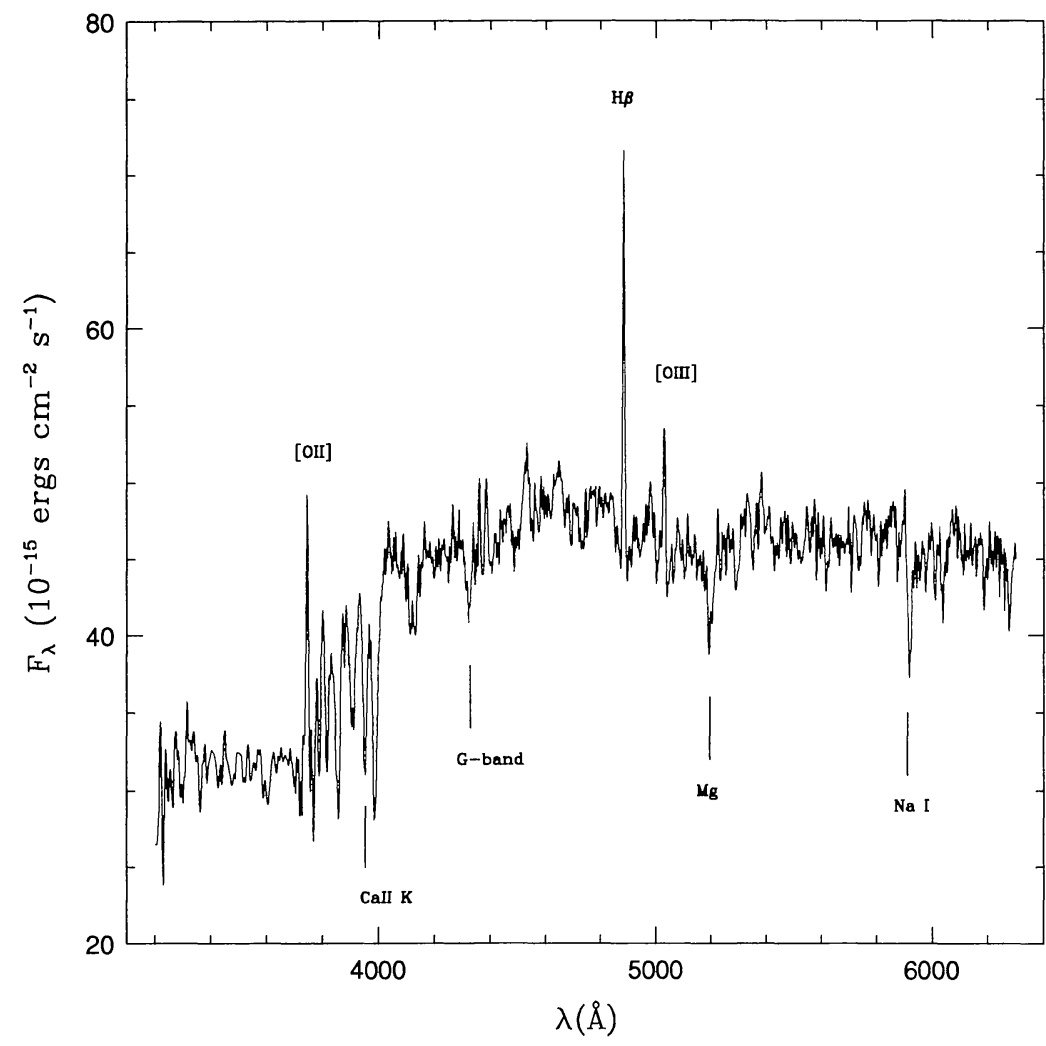

FIG. $2 a$

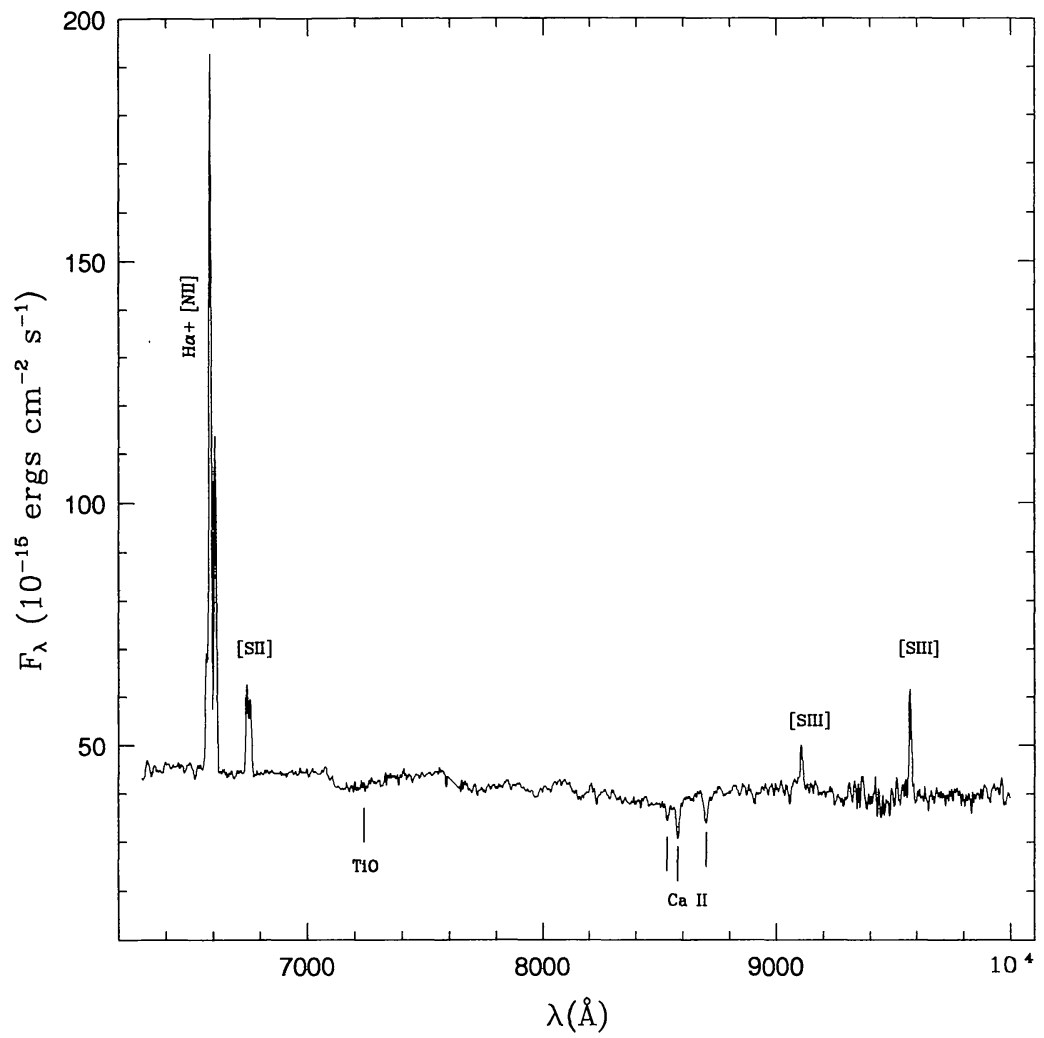

FIG. $2 b$

FIG. 2.-( $a$ ) Optical and $(b)$ near-IR spectrum of the galaxy NGC 1672. The main measured spectral features are identified.

107 


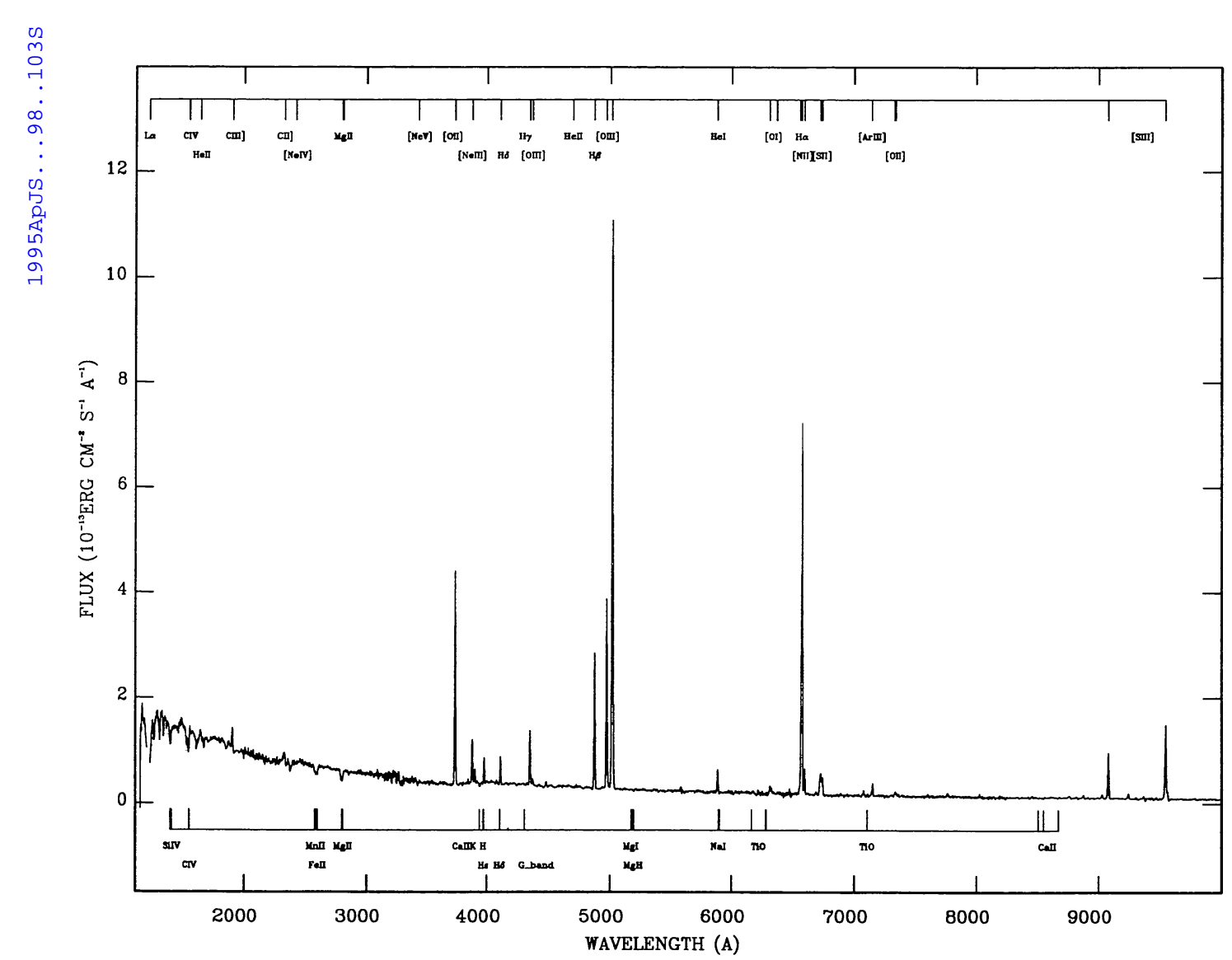

FIG. $3 a$

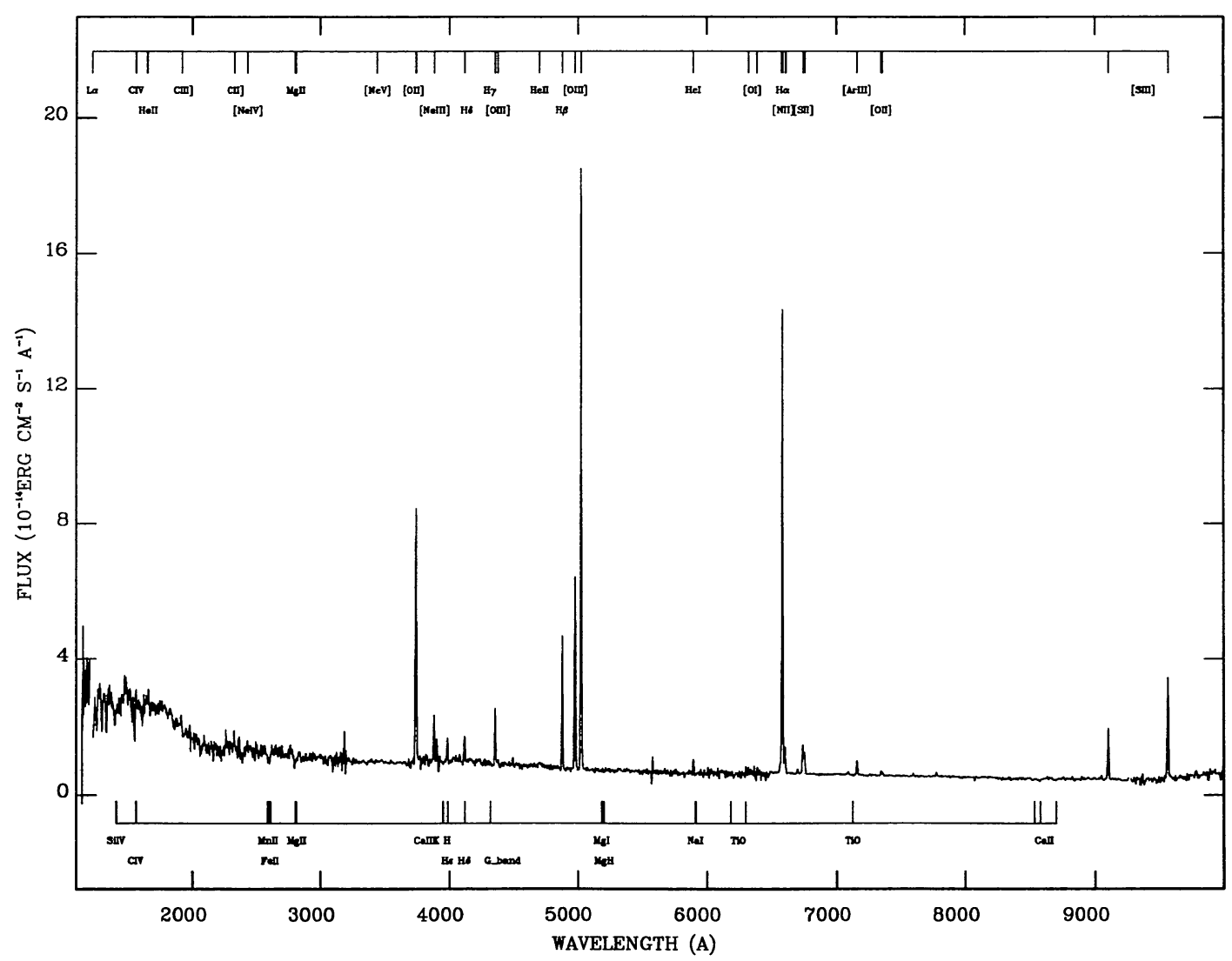

FIG. $3 b$

FIG. 3.-Spectral distributions of three BCGDs. (a) NGC 5253, (b) NGC 3125, (c) NGC 1705.

108 


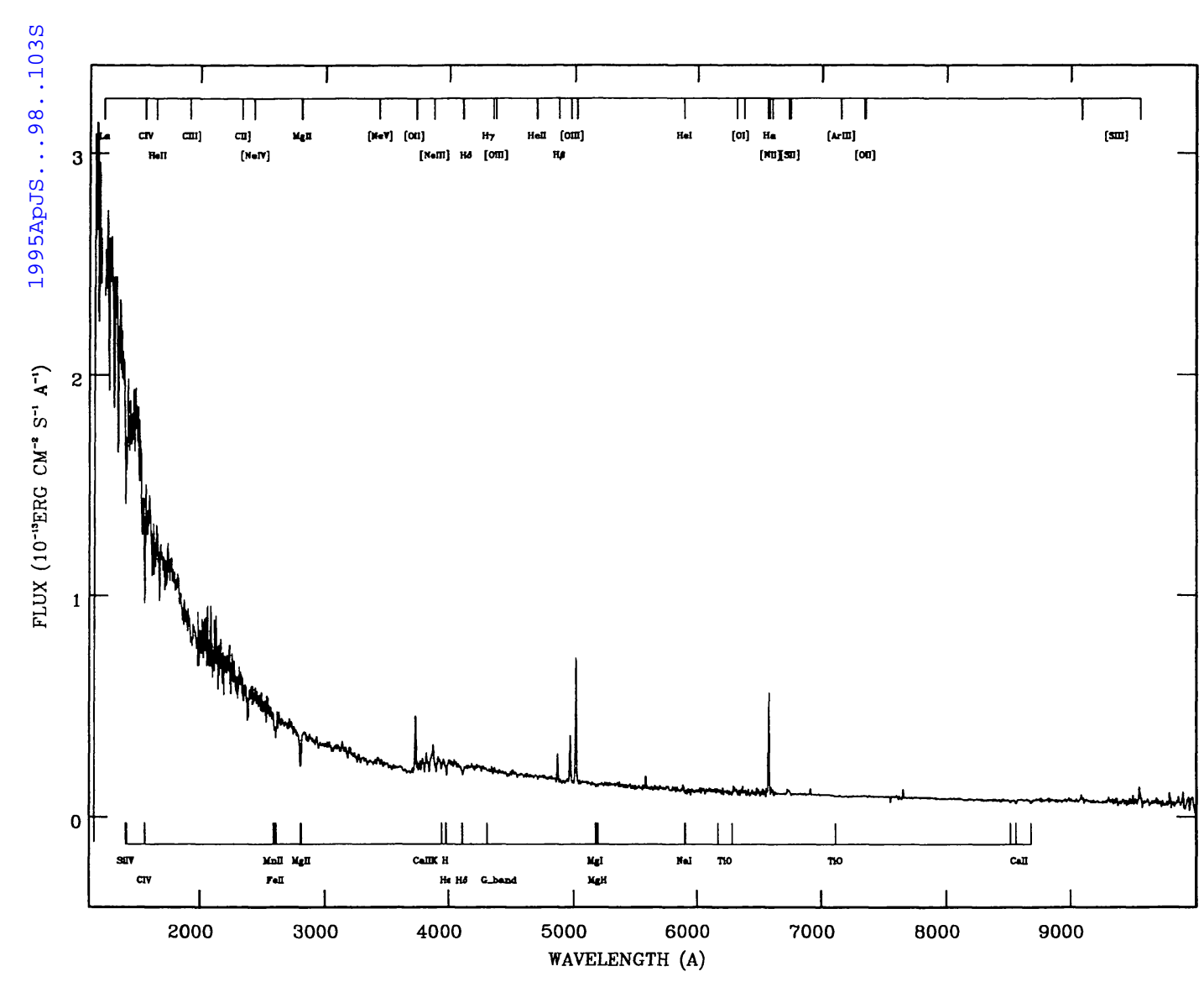

FIG. $3 c$ 


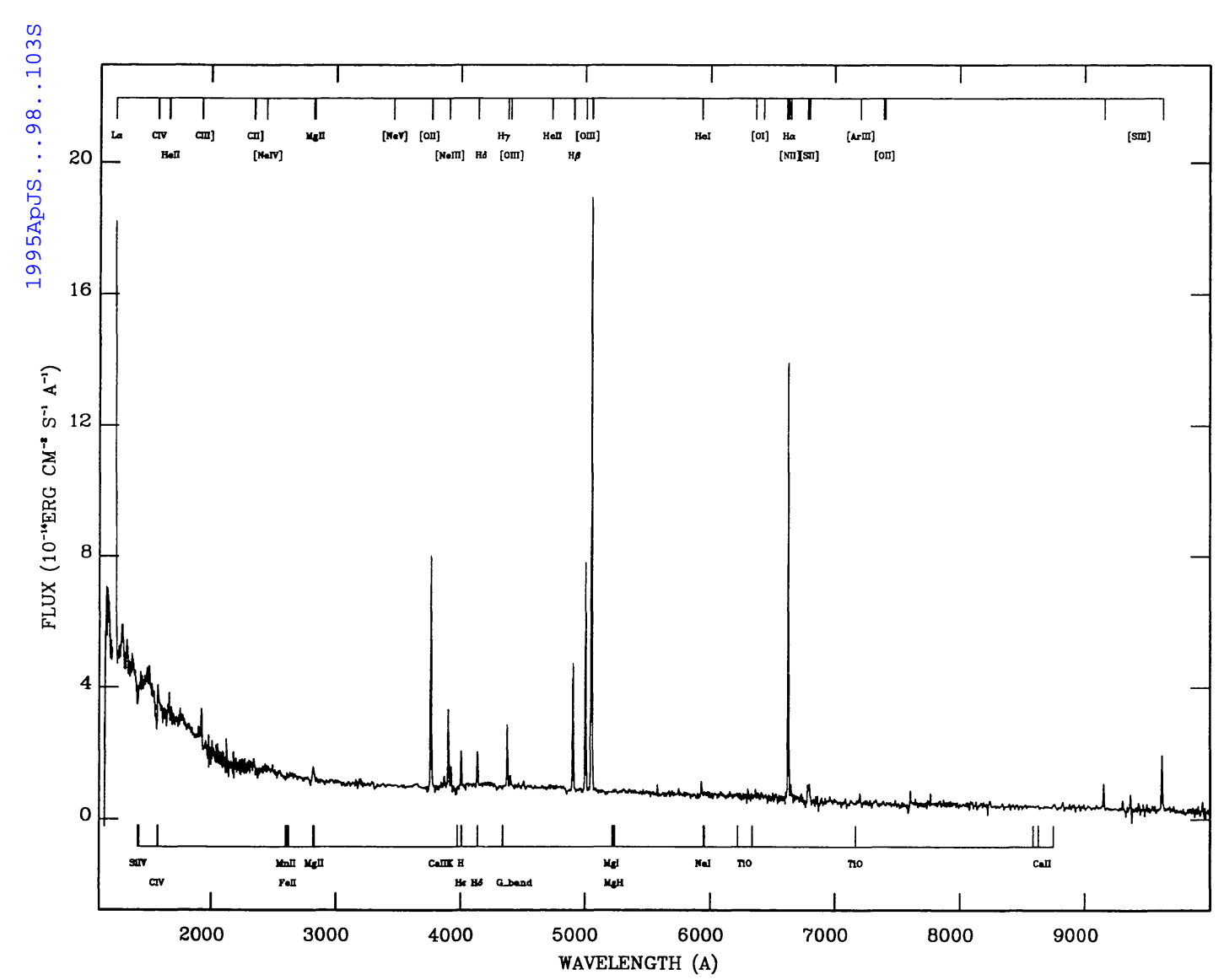

FIG. $4 a$

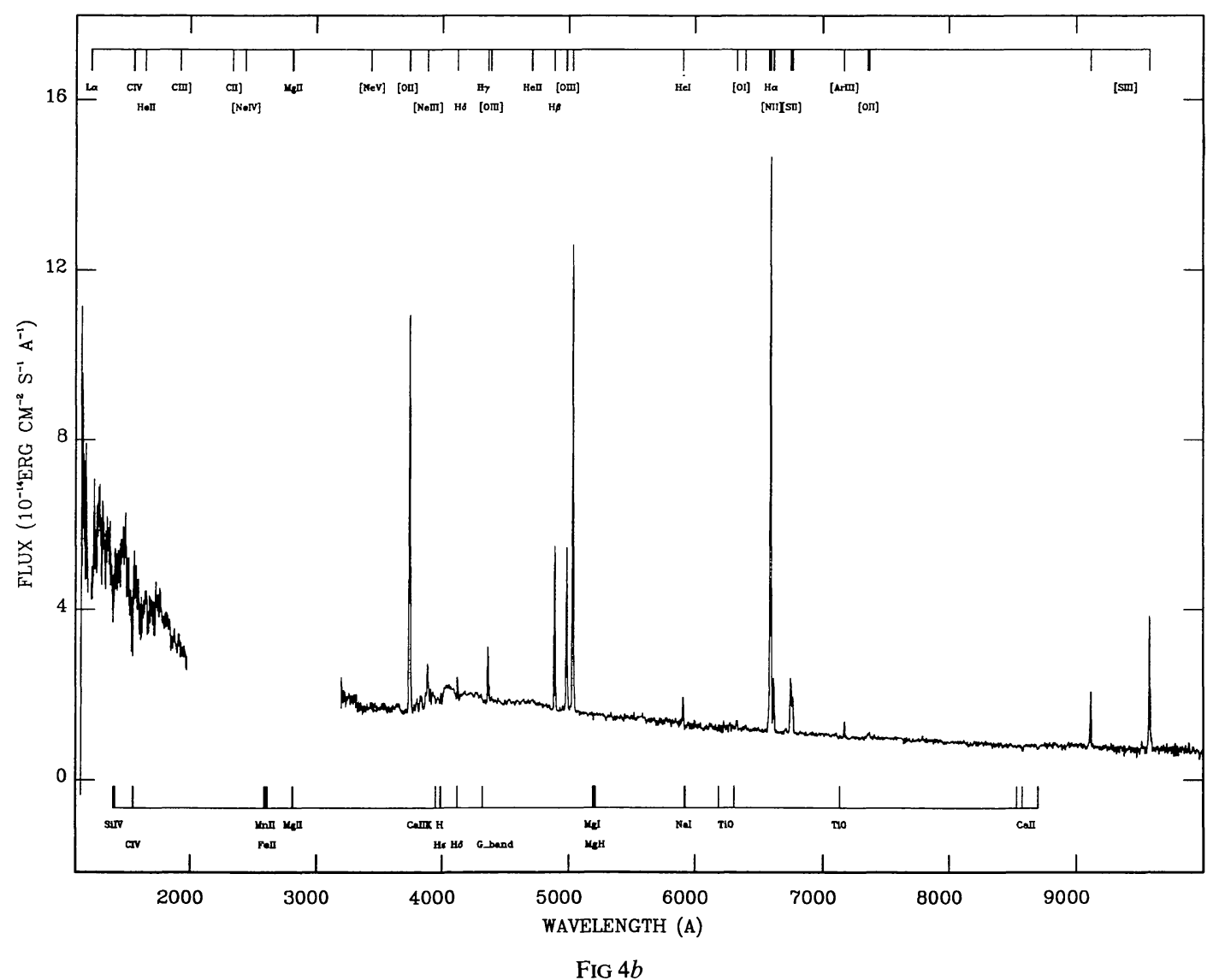

FIG. 4.-Spectra of three BCGs. ( $a$ ) TOL 1924-416, (b) NGC 1140, (c) $1050+04$. 


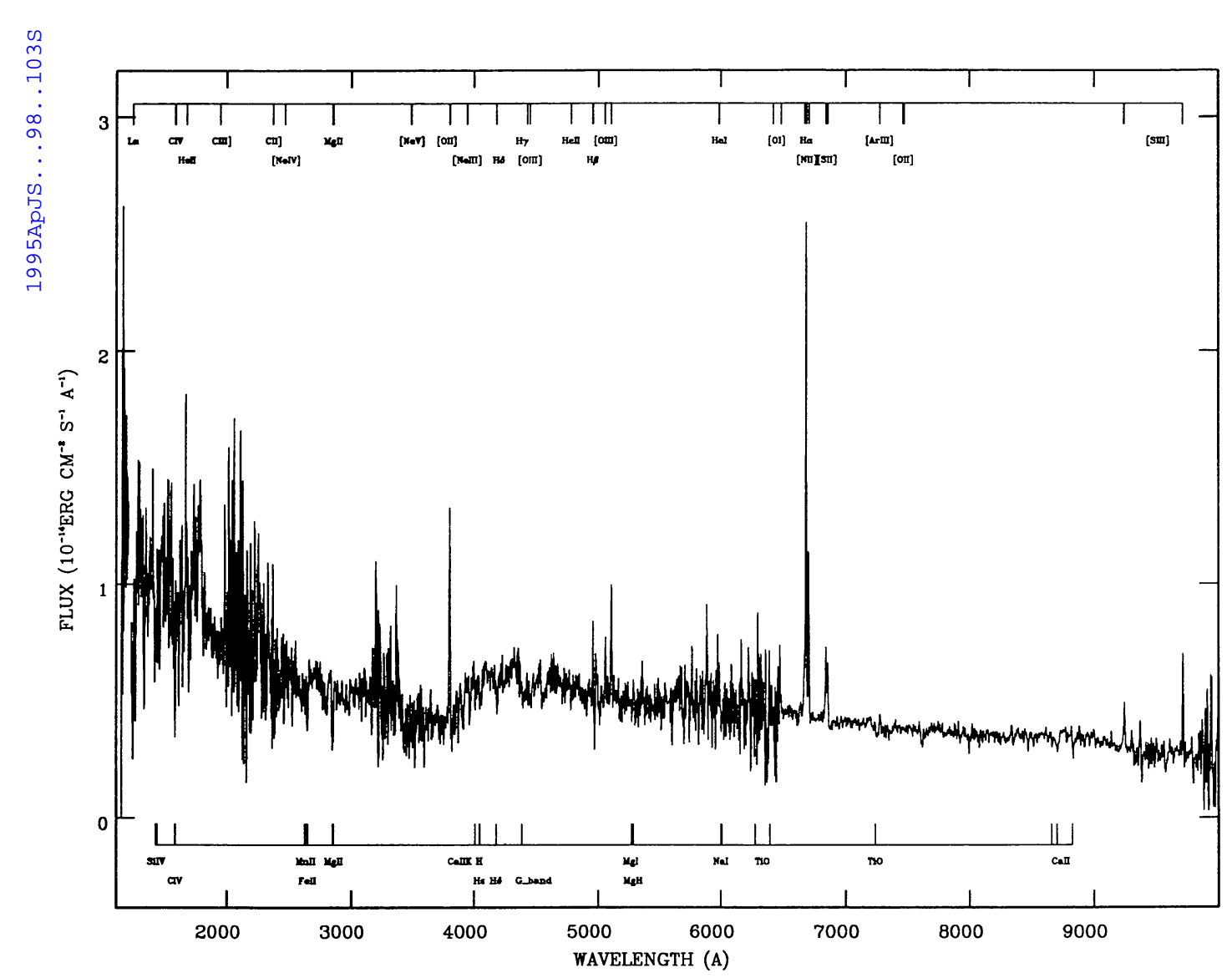

FIG. $4 c$ 


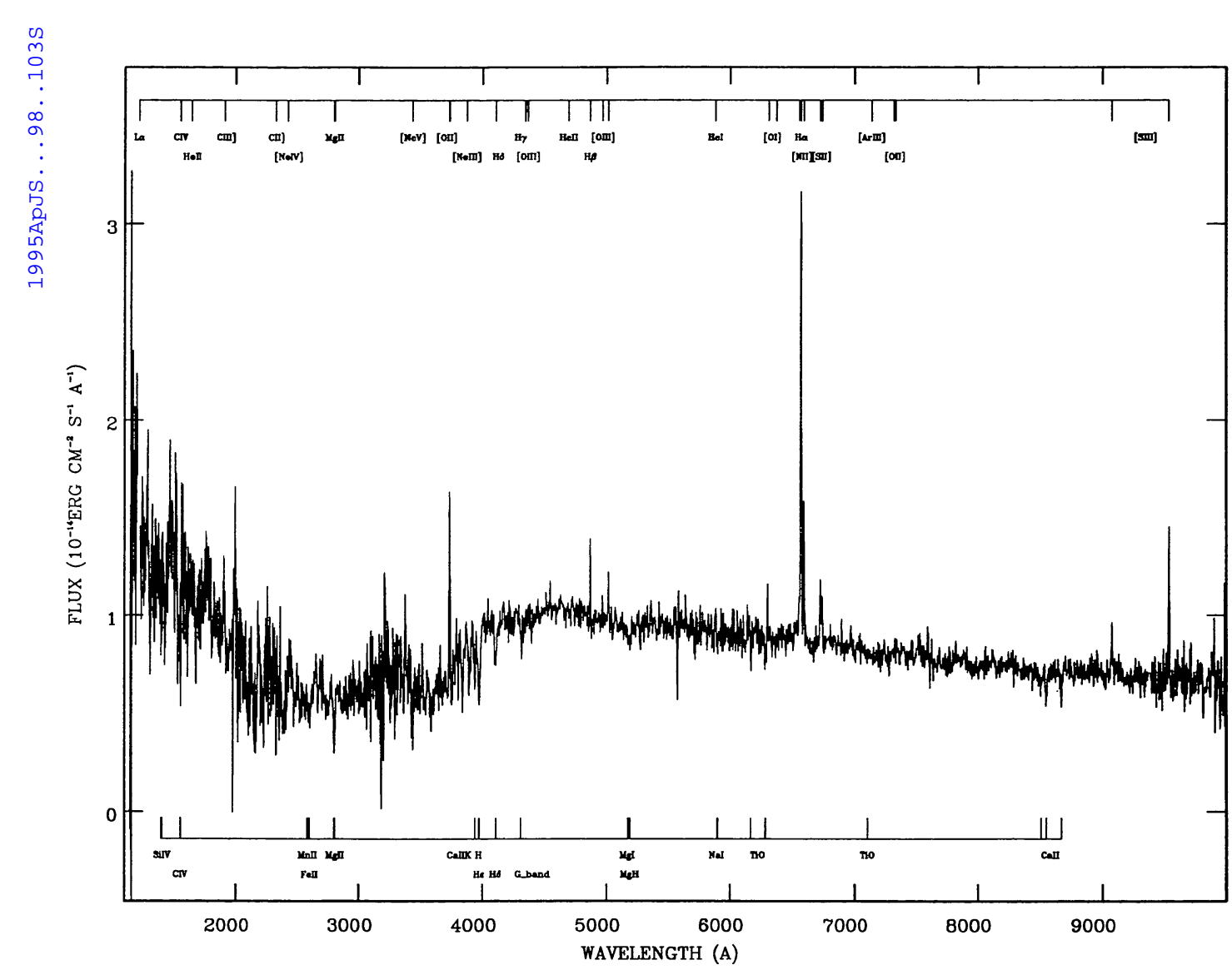

FIG. $5 a$

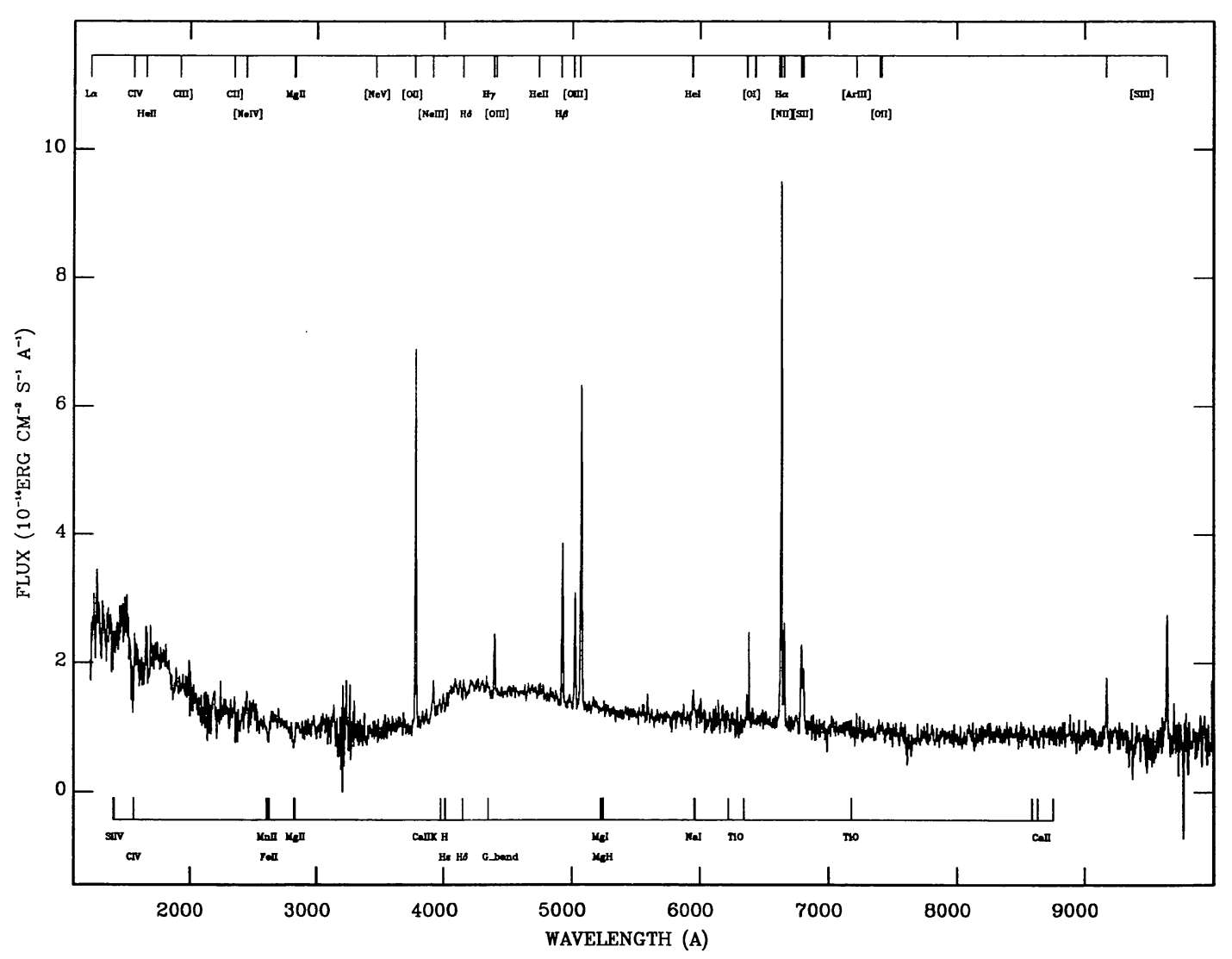

FIG. $5 b$

FIG. 5.-Spectra of two H II galaxies. ( $a$ ) NGC 7793, (b) NGC 7673. 


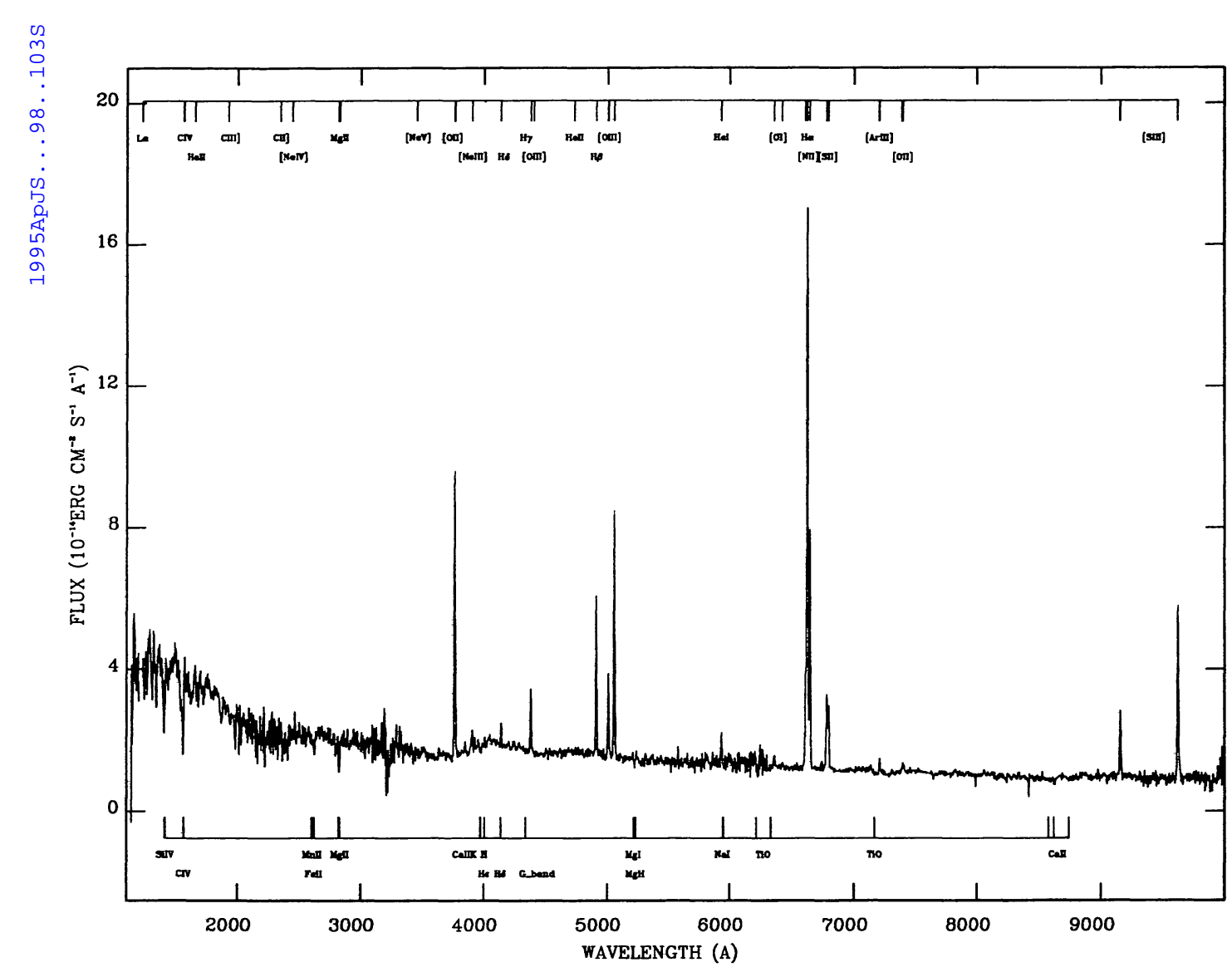

FIG. $6 a$

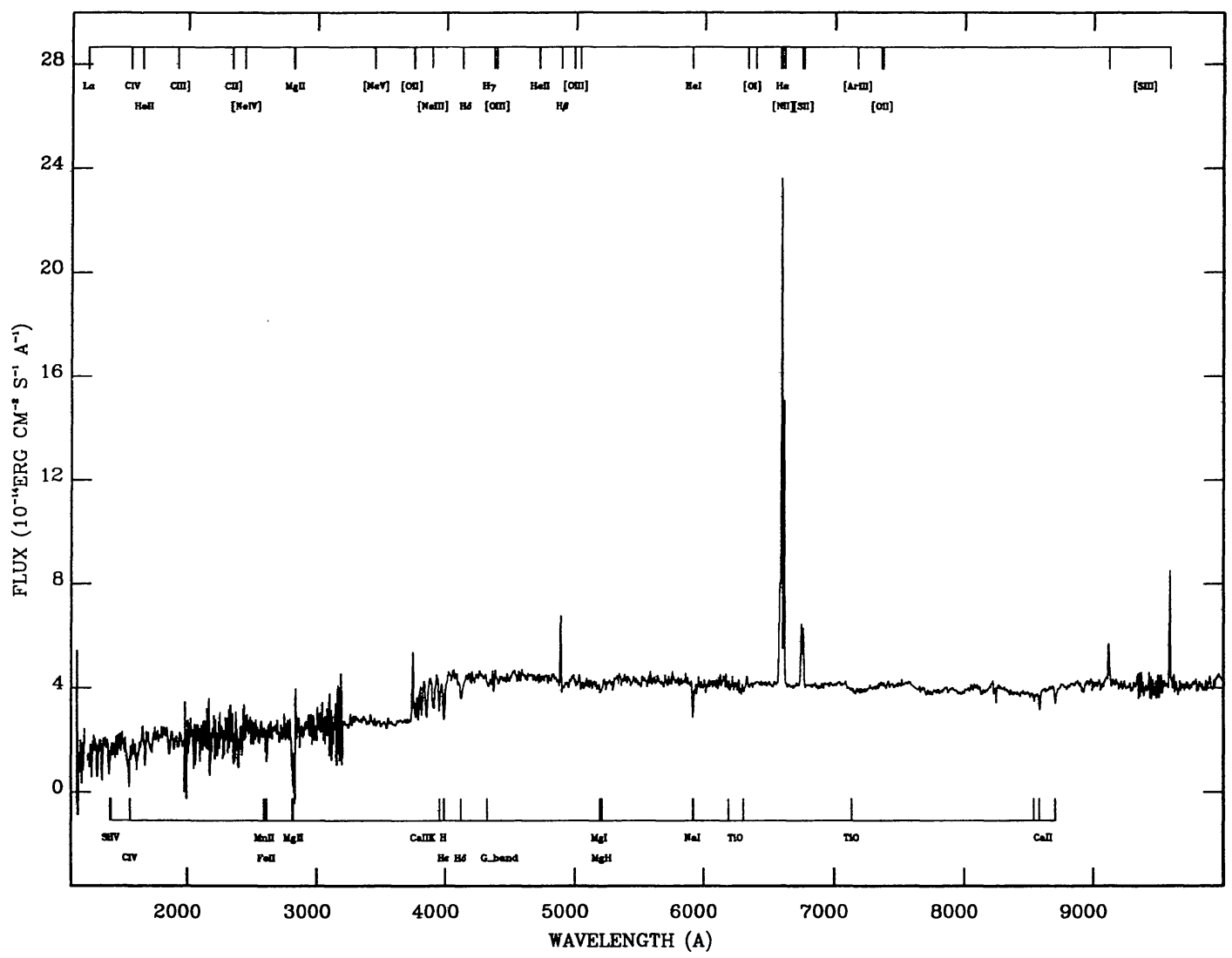

FIG. $6 b$

FIG. 6.-Spectral distributions of three starbursts. (a) NGC 7714, (b) NGC 7552, (c) NGC 5236. 


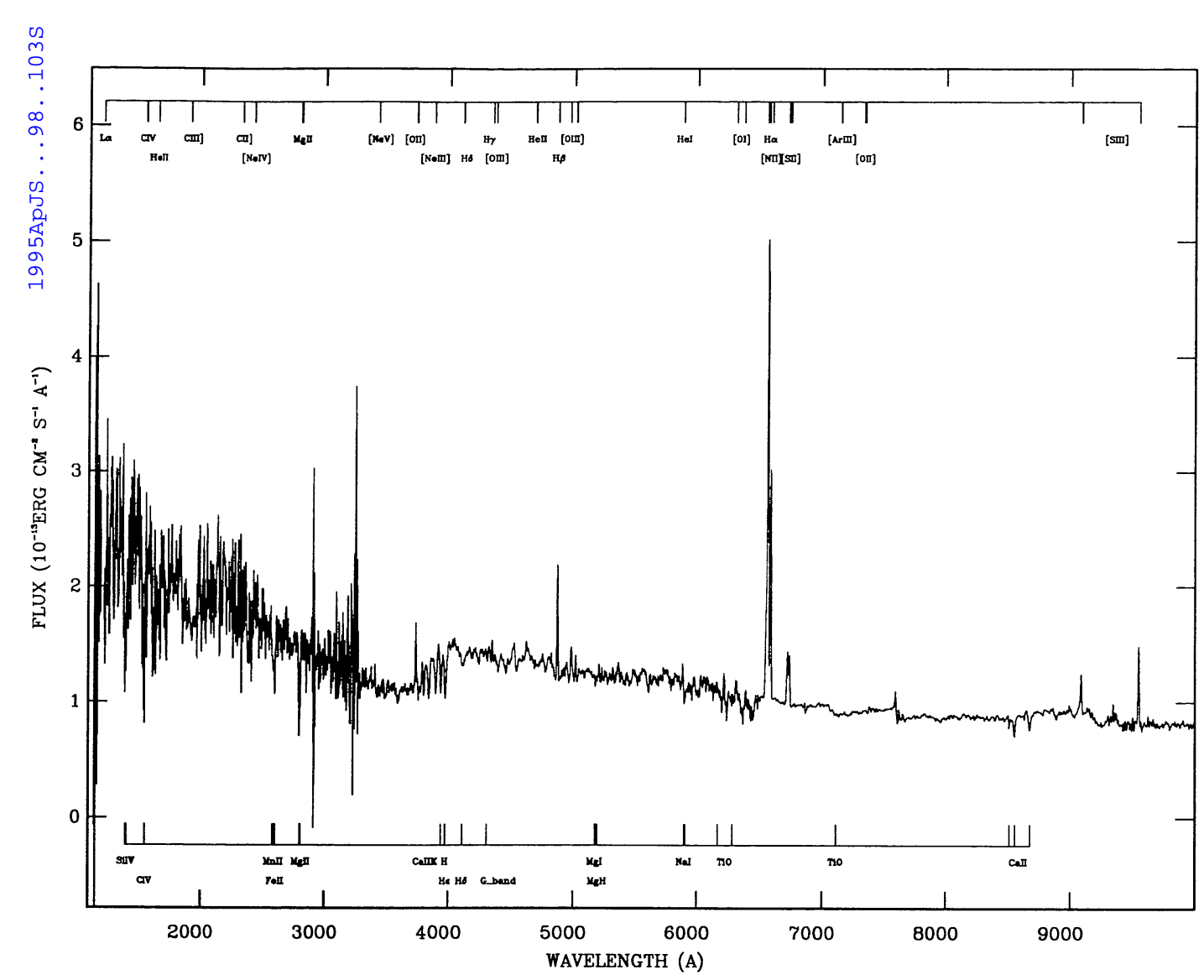

FIG. $6 c$ 


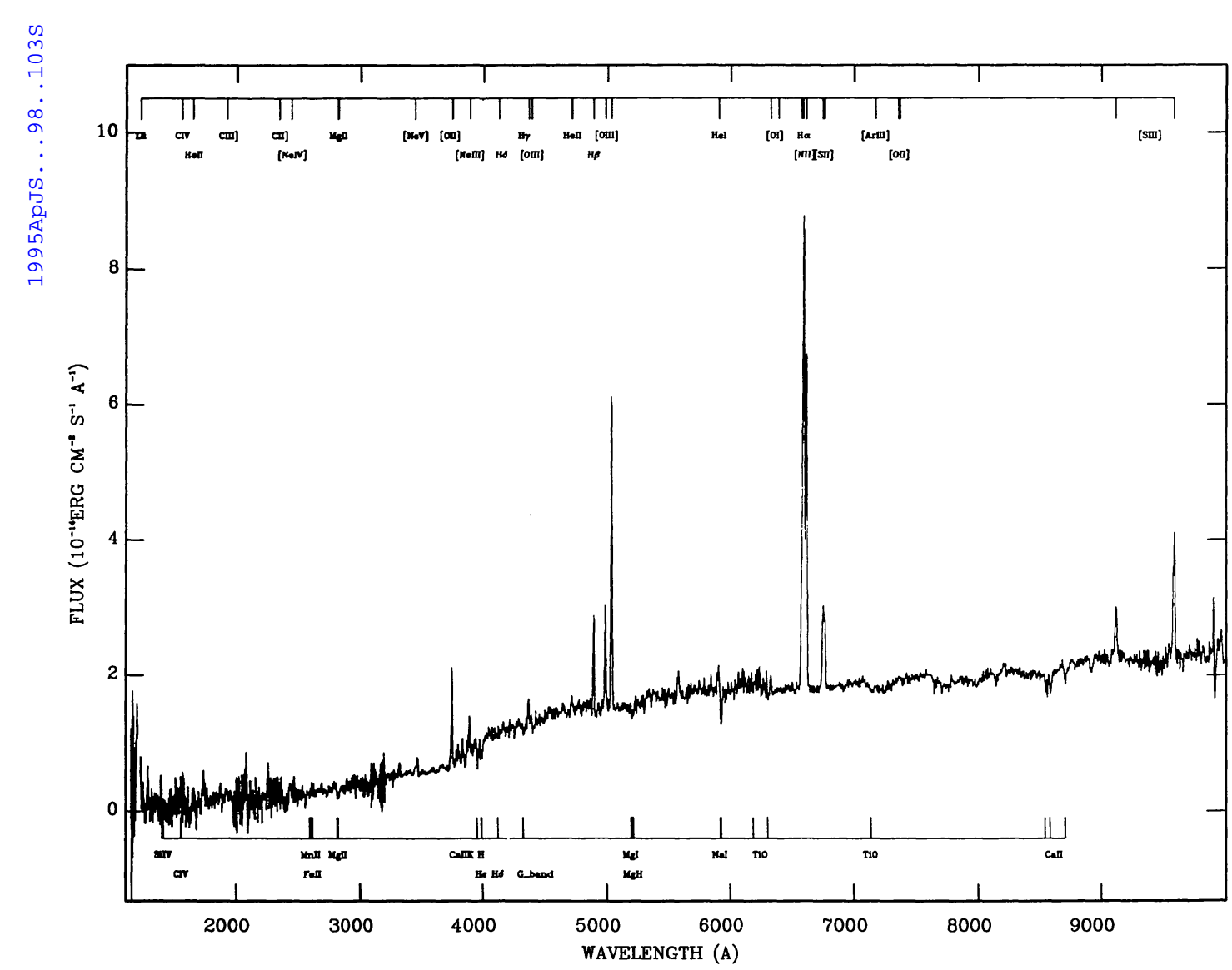

FIG. $7 a$

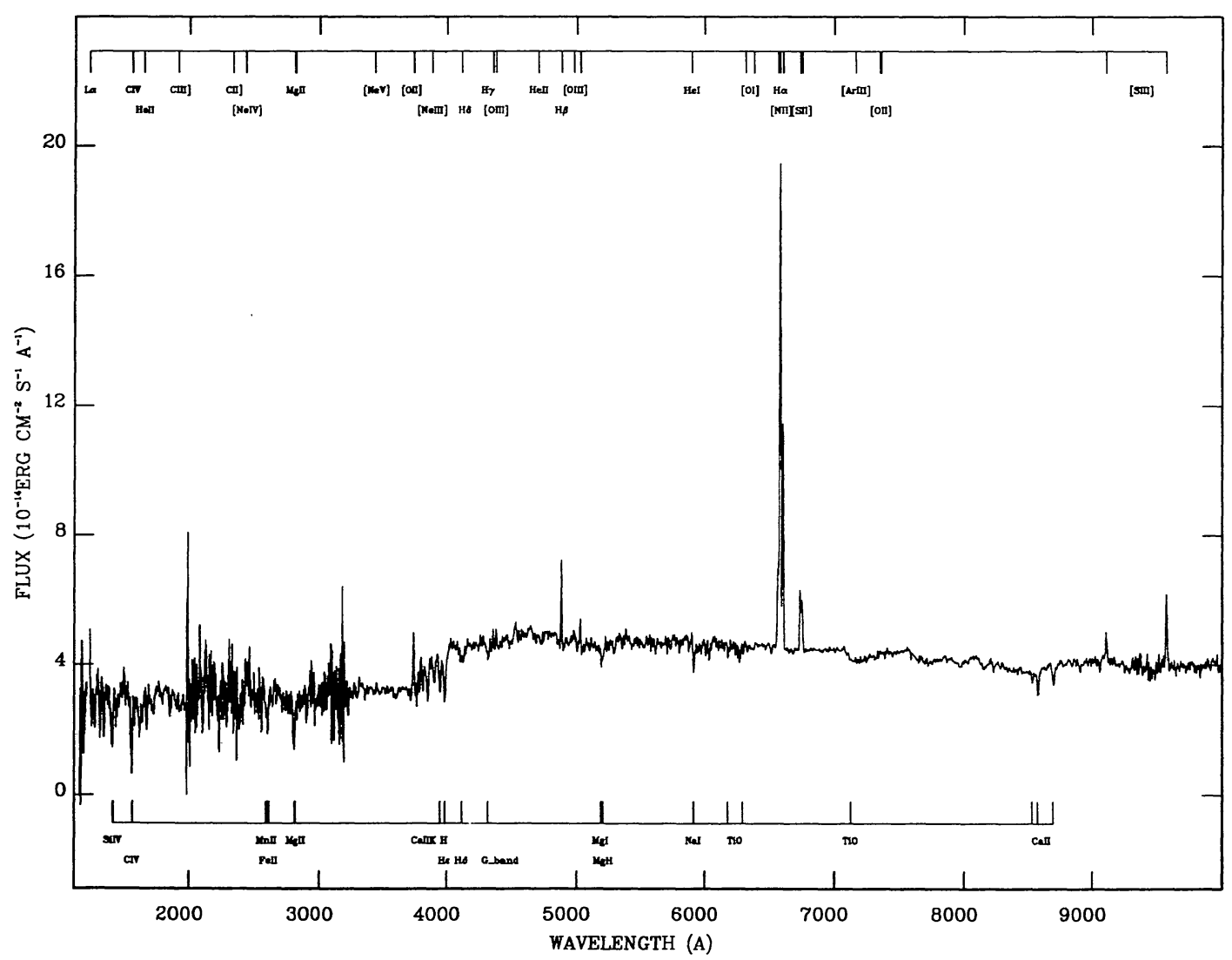

FIG. $7 b$

FIG. 7.-Spectral distributions from two composite-type galaxies. (a) NGC 7582, (b) NGC 1672. 


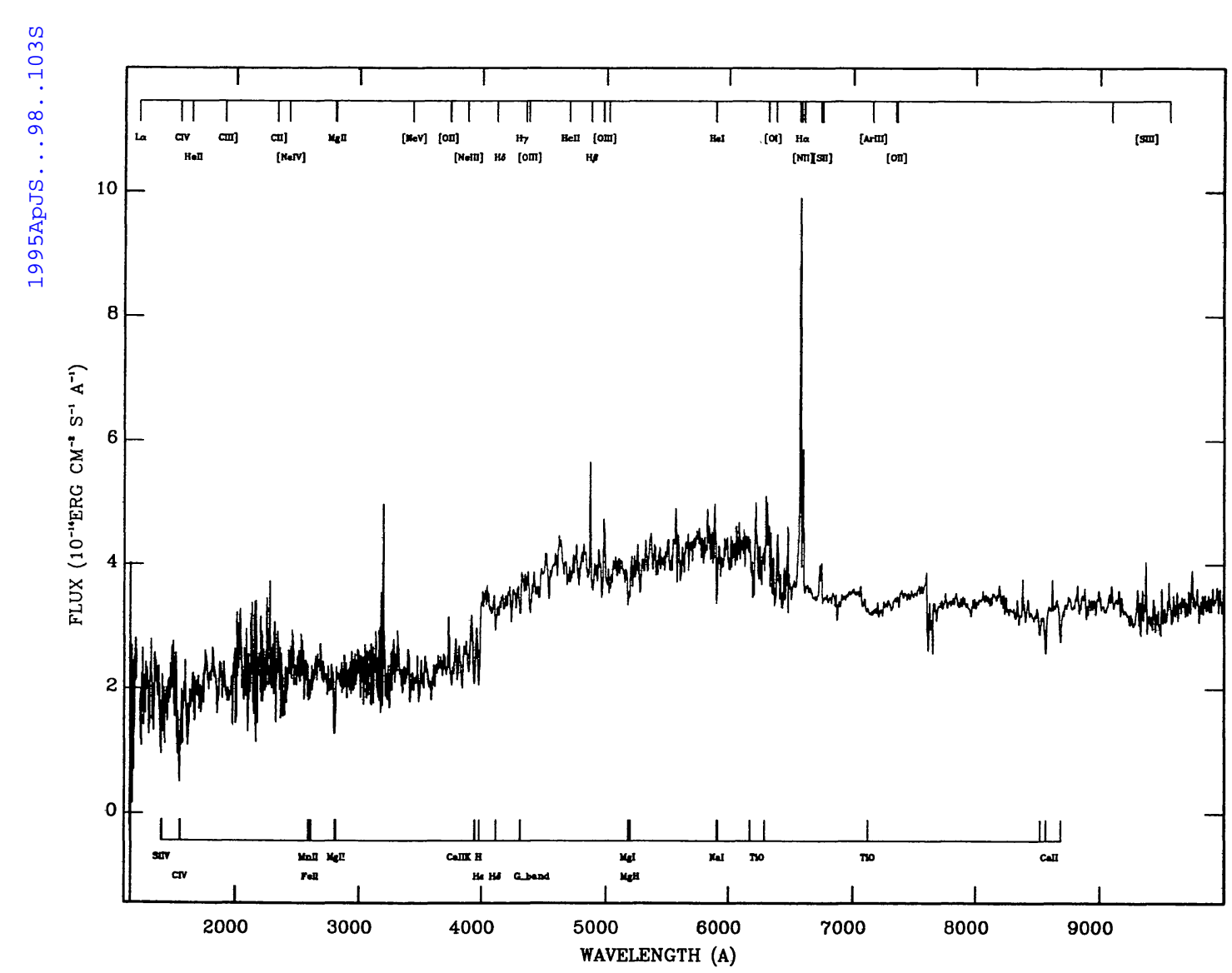

FIG. $8 a$

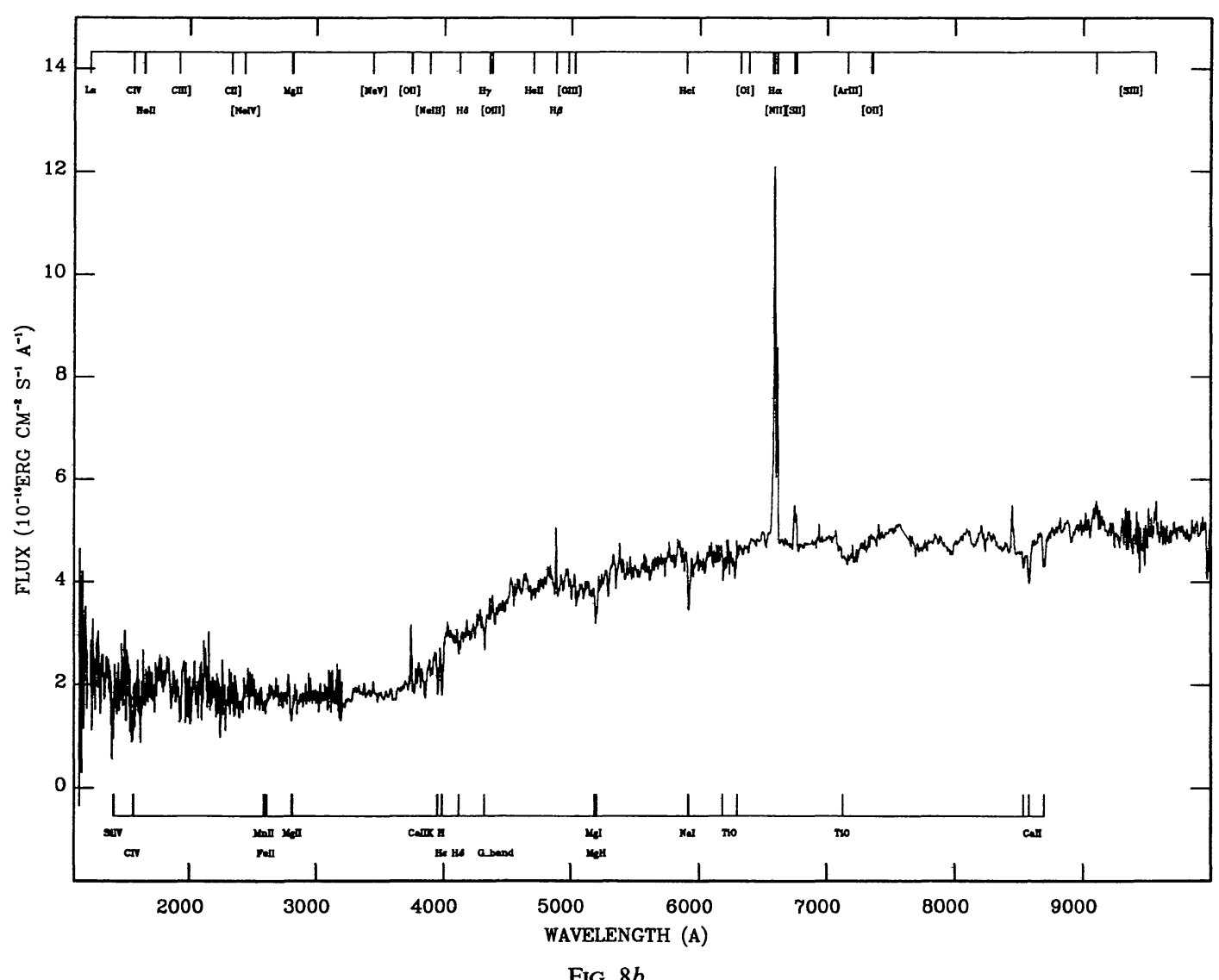

FIG. 8.-Spectra of the two hot spot galaxies. (a) NGC 3351, (b) NGC 1097. 


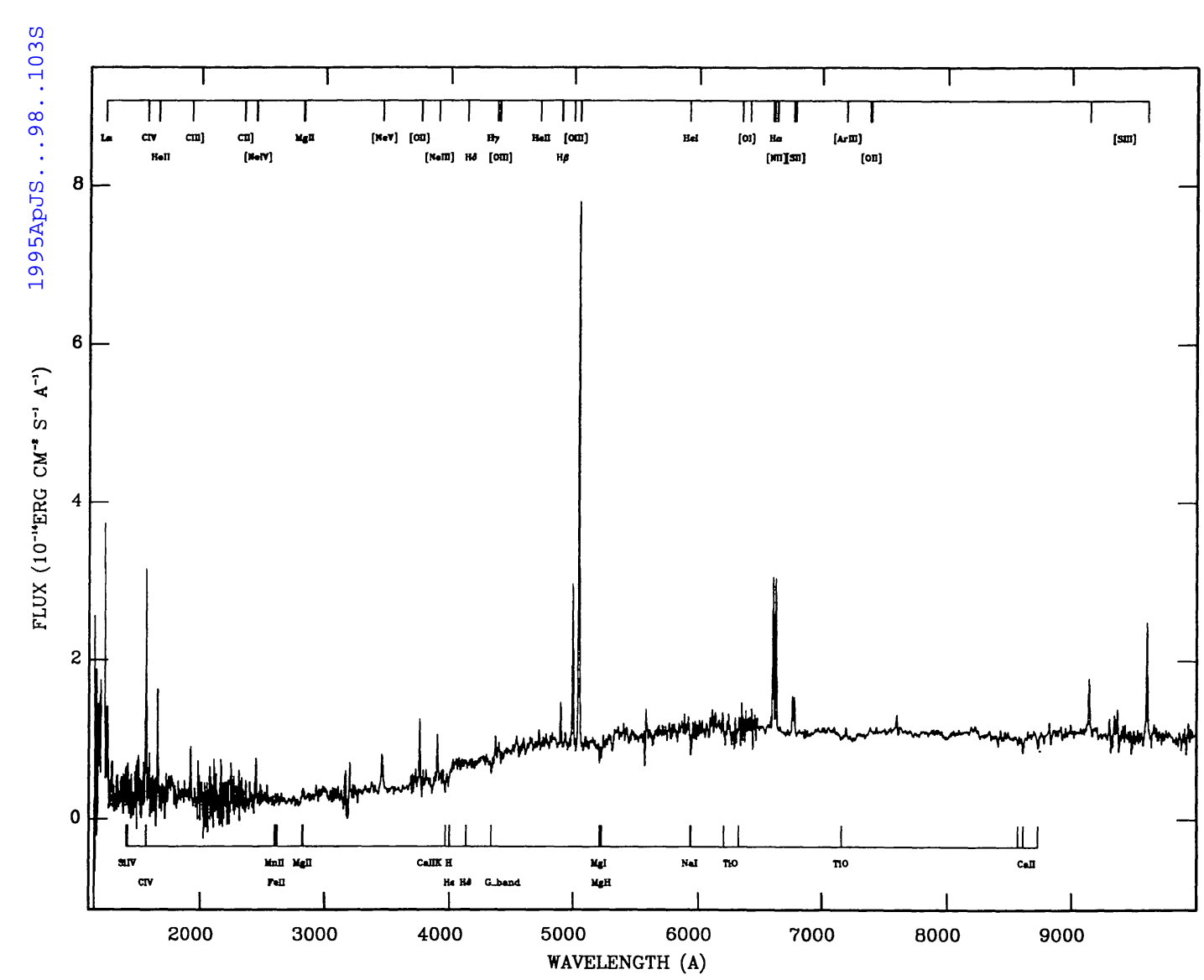

FIG. $9 a$

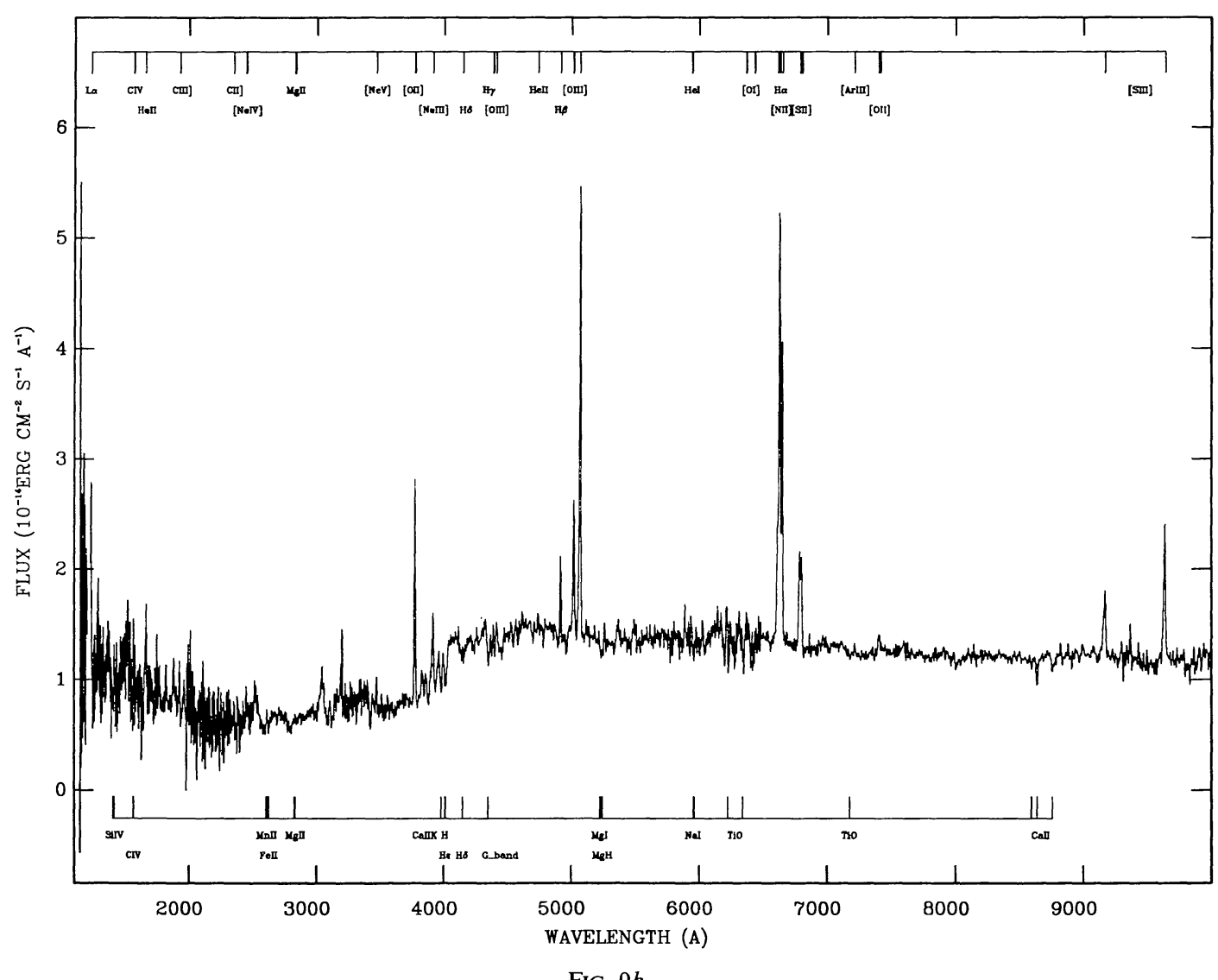

FIG. $9 b$

FIG. 9.-Spectra of two Seyfert 2 galaxies. ( $a$ ) NGC 3081, (b) IC 3639. 


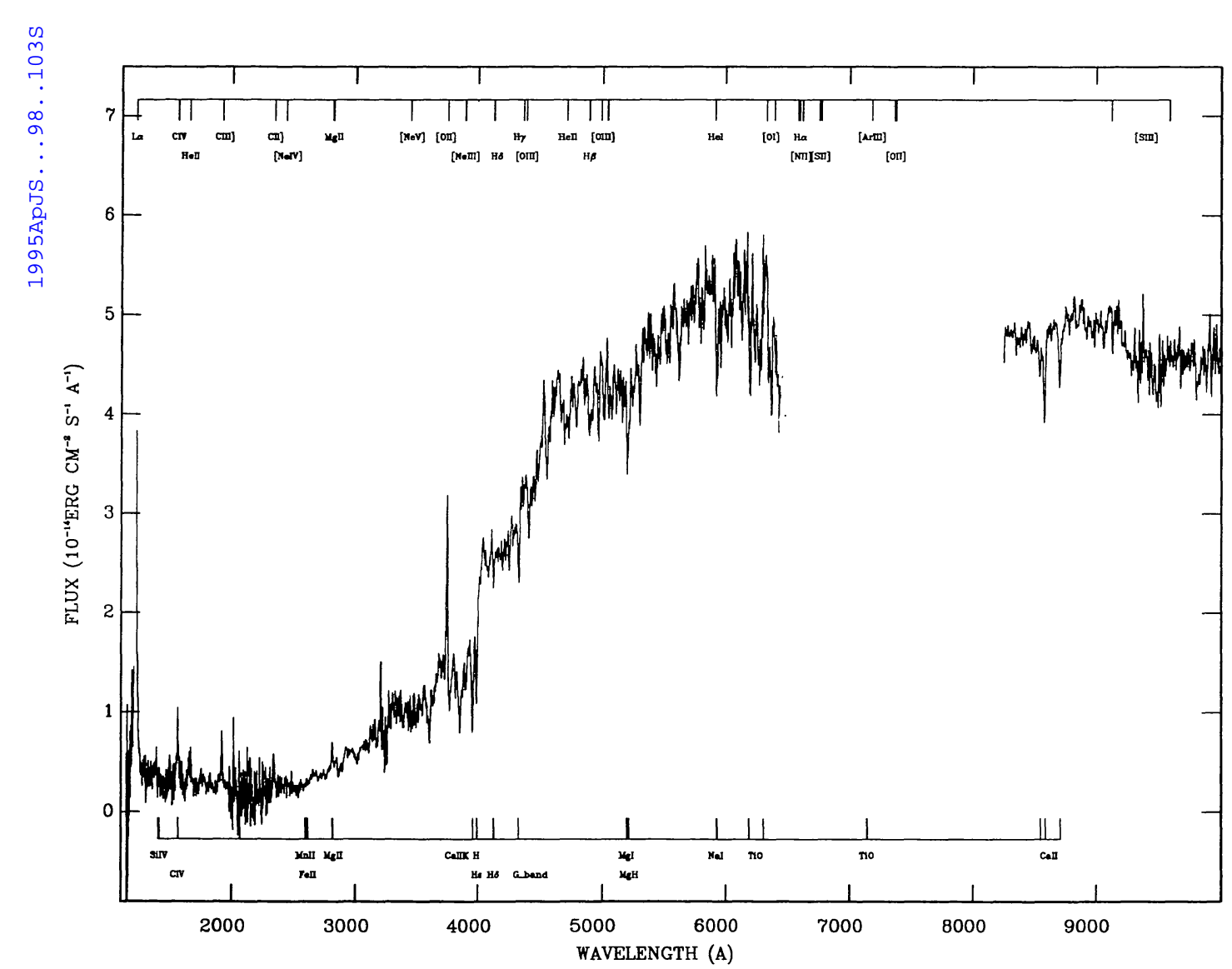

FIG. $10 a$

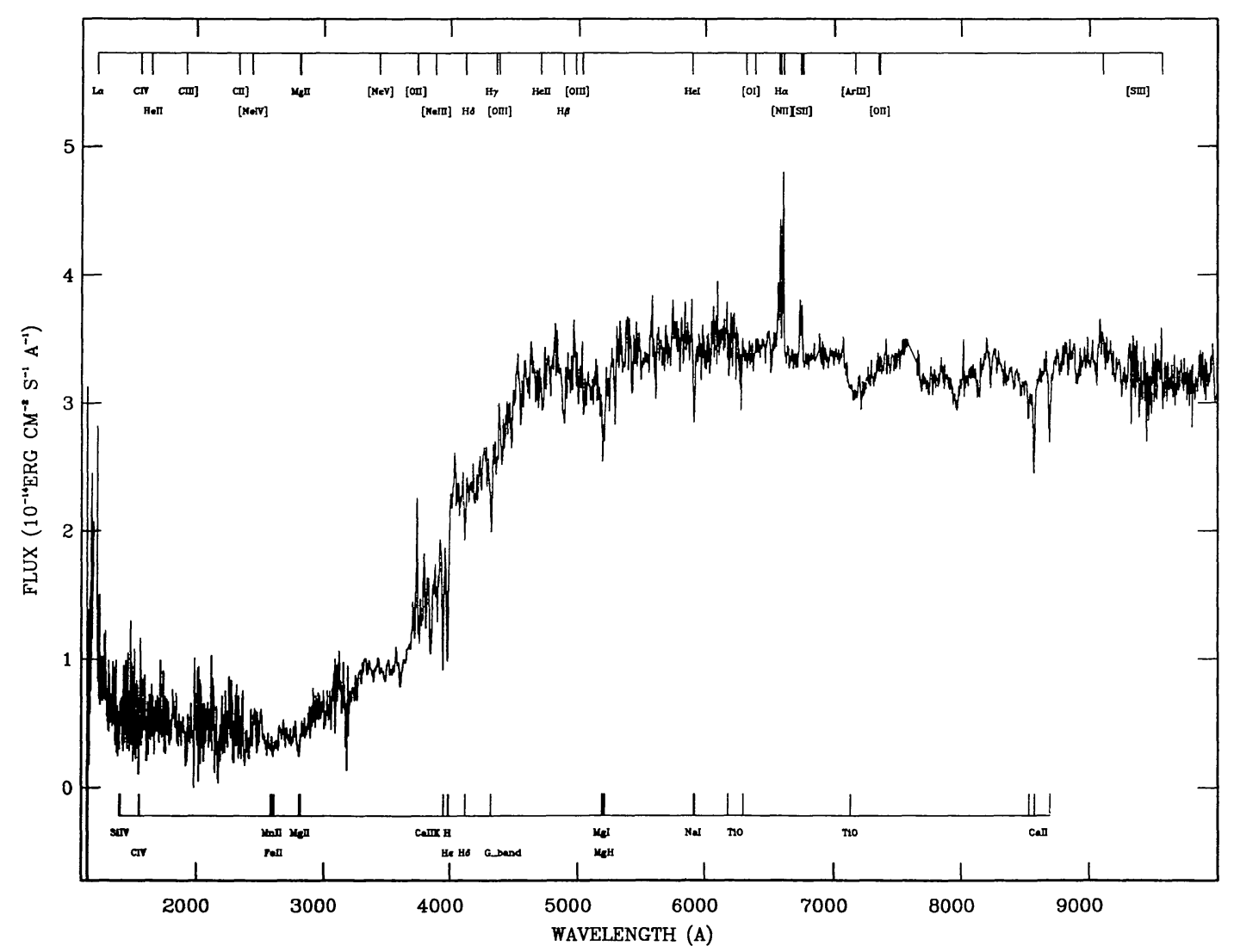

FIG. $10 b$

FIG. 10.- Spectra of two LINER galaxies. ( $a$ ) NGC 4579, (b) NGC 1433. 
TABLE 2

MEASUREd EMISSION LiNeS

\begin{tabular}{|c|c|}
\hline Line Identification & $\begin{array}{l}\text { Wavelength } \\
\text { (A) }\end{array}$ \\
\hline 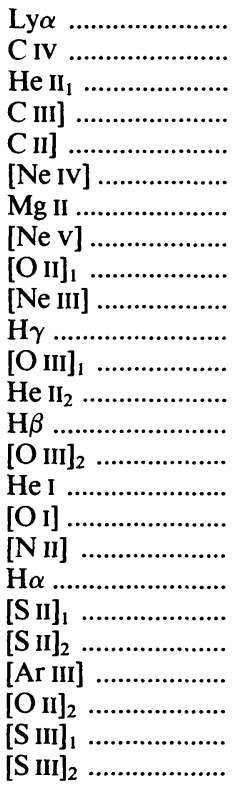 & $\begin{array}{l}1216 \\
1548,1550 \\
1640 \\
1908 \\
2326 \\
2424 \\
2796,2803 \\
3426 \\
3726,3729 \\
3869 \\
4340 \\
4363 \\
4686 \\
4861 \\
5007 \\
5876 \\
6300 \\
6584 \\
6563 \\
6717 \\
6731 \\
7135 \\
7319,7330 \\
9069 \\
9532\end{array}$ \\
\hline
\end{tabular}

Figures $1 a$ and $2 a$ show the range $\lambda \lambda 3200-6400$ and Figures $1 b$ and $2 b$ the range $\lambda \lambda 6400-10000$ for the same galaxies.

The different segments of the spectra were then combined, together with the UV spectra (Kinney et al. 1993), in order to construct spectral distributions from $\lambda 1100$ to $\lambda 10000$. The flux levels of the different segments agreeded with each other usually within $10 \%-20 \%$ in the overlapping regions. In a few cases, the differences between the flux levels were larger, mainly when the observations were made under nonphotometric conditions. In these cases, as the UV fluxes should be the most accurate (there is no atmosphere up there in the $I U E !)$, before making the measurements, we scaled the optical data to the level indicated by the flux level of the end of the UV spectrum $($ at $\lambda \approx 3200 \AA)$. The spectral distributions of the starbursts NGC 7714 (Fig. 6a) and NGC 7552 (Fig. 6b), of the composite-type galaxy NGC 7582 (Fig. 7a) and of the Seyfert 2 NGC 3081 (Fig. 9a) are examples for which no scaling was necessary between the different segments of the spectra.

\section{RESULTS}

A sample of the resulting spectral distributions are shown in Figures 3-10. A horizontal bar is drawn at the top of each plot with the location of typical emission lines marked at the redshift of the galaxies. The corresponding rest wavelengths of the lines are shown in Table 2. Beneath the spectrum, another bar shows the location of the main absorption features, whose corresponding windows are listed in Table 3. At least two representative galaxies for each activity class are shown in the figures. We have kept the classification as blue compact dwarf galaxy (BCDG), blue compact galaxy (BCG), HII, starburst
(SB), Seyfert (Sy), LINER, composite, and hot spot (Hs) as given by Kinney et al. (1993).

After bringing the spectra to the rest frame, using the radial velocities listed in Table 1 of Kinney et al. (1993), we have measured the fluxes at selected points of the continuum, which have been used in the calculation of equivalent widths of absorption lines and continuum colors. The wavelengths and fluxes are listed in Table 4, together with an estimate of the error for each region of the spectra, calculated as the rms deviation from the average continuum flux at selected wavelengths (the same error estimate can be adopted for neighboring $\lambda$ 's for which the $\mathrm{S} / \mathrm{N}$ ratio of the spectra do not change significantly). The criteria used in the wavelength selection and determination of the continuum fluxes were the following: for $\lambda 1355, \lambda 1455, \lambda 1507$, and $\lambda 1583$, we used averages in the windows, respectively, $\lambda \lambda 1348-1365, \lambda \lambda 1435-1475, \lambda \lambda 1500$ $1515, \lambda \lambda 1565-1600$. These were the spectral regions used by Robert, Leitherer, \& Heckman (1993) for determining the continuum for the calculation of the equivalent widths of the absorption lines of Si IV $\lambda 1400$ and C IV $\lambda 1550$. We decided to this because we have used the results of their work in the analysis of our data. For the region between 2000 and $3500 \AA$ we present the average continuum fluxes in $15 \AA$ bins centered on the wavelengths $\lambda 2530$ and $\lambda 2900$, selected because the neighboring spectral regions are free from prominent spectral features. The other continuum wavelengths (in the visual and near-IR) were kindly suggested by E. Bica (see Bica 1988), and most of them sample regions free from emission and absorption lines. The corresponding fluxes were determined as averages in $15 \AA$ bins centered in the listed wavelengths, except for the values corresponding to $\lambda 6563(\mathrm{H} \alpha)$, which was a visual estimate of the continuum at the base of the line, and $\lambda 3810$ or $\lambda 3780$, which were also visual estimates. These last points were necessary for the determination of the continuum in galaxies with strong contribution of late B to F stars which present several high-order Balmer absorption lines in this region. Due to the crowding of the absorption lines, it is difficult to make automatic measurements. We thus selected the higher of the $\lambda 3810$ and $\lambda 3780$ fluxes to represent the continuum in this spectral region.

TABLE 3

MEASURED ABSORPTION FEATURES

\begin{tabular}{|c|c|c|}
\hline $\begin{array}{l}\text { Window } \\
(\AA)\end{array}$ & $\begin{array}{c}\text { Main } \\
\text { Absorber }\end{array}$ & Identification \\
\hline $1380-1415 \ldots \ldots$ & Si IV & Si IV \\
\hline $1523-1576 \ldots \ldots . .$. & C IV & C IV \\
\hline 2570-2615 ......... & $\mathrm{Fe}$ II & $\mathrm{Fe}$ II \\
\hline $2780-2825 \ldots \ldots . .$. & $\mathrm{Mg}_{\text {II }}$ & $\mathrm{Mg}_{\text {II }}$ \\
\hline 3908-3952 ......... & $\mathrm{Ca}$ II K & $\mathrm{Ca} \mathrm{K}$ \\
\hline 3952-3988 ......... & $\mathrm{Ca}$ II $\mathrm{H}+\mathrm{H} \epsilon$ & $\mathrm{CaH}$ \\
\hline $4150-4214 \ldots \ldots$ & $\mathrm{CN}$ & $\mathrm{CN}$ \\
\hline 4284-4318 ......... & $G$ band & $G$ band \\
\hline 5156-5196 ......... & $\mathrm{Mg} \mathrm{I}+\mathrm{MgH}$ & $\mathrm{Mg}$ \\
\hline 5880-5914 ......... & $\mathrm{Na} I$ & $\mathrm{NaI}$ \\
\hline 7158-7274 .......... & $\mathrm{TiO}$ & $\mathrm{TiO}$ \\
\hline $8476-8520 \ldots \ldots .$. & Ca II & $\mathrm{Ca}$ II (1) \\
\hline 8520-8564 ......... & Ca II & $\mathrm{Ca}$ II (2) \\
\hline $8640-8700 \ldots \ldots .$. & Ca II & $\mathrm{Ca}$ II (3) \\
\hline
\end{tabular}


TABLE 4

AVERAge FluXes at Selected ContinuUM WAVELENGTHS ${ }^{1}$

Wavelengths in $\AA$

galaxy

\begin{tabular}{lllllllllllllllllllll}
1355 & 1455 & 1507 & 1583 & 2530 & 2900 & 3500 & 3740 & 3810 & 4020 & 4510 & 4630 & 5313 & 5870 & 6080 & 6563 & 7043 & 7525 & 8180 & 8838 \\
\hline
\end{tabular}

HARO15

$\begin{array}{lllllllll}22.2 & 22.7 & 18.9 & 17.4 & - & - & 5.3 & 5.9\end{array}$

$\begin{array}{lllll}4810 & 4020 & 4510 & 4630 & 531\end{array}$

ESO296-11

$\begin{array}{llllll}18.9 & 3.0 & - & 0.4\end{array}$ \begin{tabular}{lllllllllll}
7.8 & 7.4 & 6.0 & 5.8 & 4.5 & 4.0 & 3.9 & 3.4 \\
\hline & & 0.3 & & 0.2
\end{tabular} 0.9

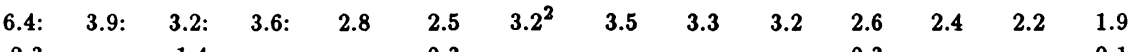
$\begin{array}{lrlrrrrrrrrrrrrrrrrrrr}\text { NGC1068 } & 63.5 & 60.2 & 61.1 & 62.8 & 45.0 & 48.9 & 64.7 & 71.4 & - & 100.8 & 122.0 & 124.8 & 138.1 & 147.1 & 143.5 & 135.6 & 134.4 & 127.3 & 134.6 & 136.5\end{array}$

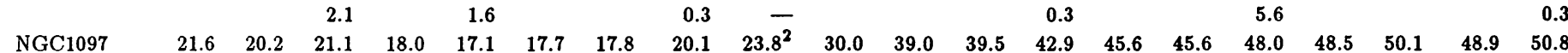

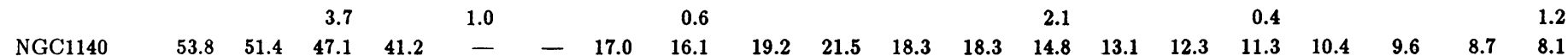

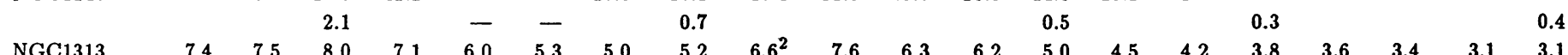
$\begin{array}{llllllllllllllllllllll}\text { NGC1313 } & 7.4 & 7.5 & 8.0 & 7.1 & 6.0 & 5.3 & 5.0 & 5.2 & 6.6^{2} & 7.6 & 6.3 & 6.2 & 5.0 & 4.5 & 4.2 & 3.8 & 3.6 & 3.4 & 3.1 & 3.1 \\ & & & 1.0 & & 0.5 & & & 0.3 & & & & & 0.2 & & & 0.1 & & & & 0.2\end{array}$

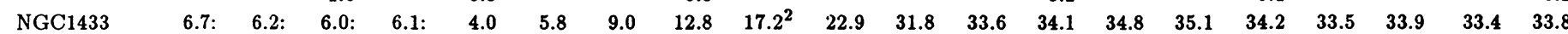
$\begin{array}{lrrrrrrrr} & & & 2.0 & & 0.4 & & & 1.3 \\ \text { NGC1510 } & 26.9 & 25.2 & 25.1 & 22.4 & 9.9 & 8.7 & 7.6 & 8.0\end{array}$ $\begin{array}{llllllllll} & & & & 2.1 & & 0.8 & & & 0.2 \\ \text { NGC1614 } & 5.8 & 6.9 & 4.5 & 5.5 & - & - & 5.6 & 5.7\end{array}$ $\begin{array}{llllllllllll}11.1 & 12.9 & 10.3 & 10.2 & 7.8 & 7.2 & 7.1 & 6.6 & 6.0 & 5.5 & - & -\end{array}$ $\begin{array}{rrrrrrrrrrrr}7.4 & 8.7 & 8.5 & 8.5 & 8.6 & 9.4 & 9.3 & 9.5 & 9.8 & 10.0 & 10.1 & 11.1\end{array}$ $\begin{array}{lrrrrrrrr}\text { NGC1672 } & 30.3 & 29.5 & 29.7 & 26.1 & 31.3 & 29.3 & 31.6 & 31.7\end{array}$ $\begin{array}{llllllllll} & & & & 1.9 & & 4.1 & & & 1.1 \\ \text { NGC1667 } & 3.4 & 3.3 & 1.8 & 2.0 & - & - & 3.9 & 4.6\end{array}$ \begin{tabular}{rrrr}
7.4 & 8.7 & 8.5 & 8.5 \\
\hline 40.9 & 45.7 & 50.5 & $50.5 \quad 47.4$
\end{tabular} 0.2 $\begin{array}{lllllllllll}45.7 & 50.5 & 50.5 & 47.4 & 46.2 & 46.6 & 44.7 & 44.4 & 44.2 & 41.0 & 41.4\end{array}$ $\begin{array}{rrrrrrrrrrrr}- & 8.3 & 11.3 & 11.4 & 11.4 & 12.6 & 12.2 & 12.1 & 12.2 & 12.5 & 12.0 & 12.6\end{array}$ $\begin{array}{ccccccc}- & 0.4 & 0.2 & 0.5\end{array}$ $\begin{array}{llllllllllll}27.7 & 24.4 & 19.9 & 18.7 & 14.7 & 12.5 & 12.0 & 10.8 & 10.0 & 9.1 & 8.1 & 7.8\end{array}$ $\begin{array}{lllllllll}\text { NGC1705 } & 210.5 & 175.1 & 161.2 & 132.2 & 47.5 & 33.8 & 23.4 & 22.1\end{array}$ $\begin{array}{lrrrrrrrr} & & & & & & & & \\ \text { NGC1800 } & 21.3 & 15.9 & 15.1 & 14.9 & - & - & 6.8 & 7.6\end{array}$

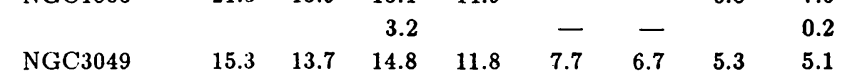
$\begin{array}{rrrrrrrrr}N G C 3049 & 15.3 & 13.7 & 14.8 & 11.8 & 7.7 & 6.7 & 5.3 & 5.1 \\ & & & 1.4 & & 0.6 & & & 0.3\end{array}$ $\begin{array}{lllllllll}\text { NGC3081 } & 3.0 & 2.6 & 2.6 & 3.1 & 2.3 & 3.0 & 3.7 & 4.6 \\ & & & 1.3 & & 0.5 & & & 0.7\end{array}$ $\begin{array}{rrrrrrrrr}\text { NGC3125 } & 27.9 & 27.8 & 27.8 & 24.5 & 12.9 & 11.1 & 9.6 & 10.1 \\ & & & 2.7 & & 1.0 & & & 0.6\end{array}$ $\begin{array}{lrrrrrrrr}\text { NGC3256 } & 13.2 & 13.4 & 13.0 & 14.0 & - & - & 13.9 & 14.2 \\ & & & 1.7 & & - & - & & 0.6\end{array}$ $\begin{array}{lrrrrrrrr}\text { NGC3351 } & 21.3 & 18.8 & 21.2 & 17.3 & 22.8 & 21.6 & 22.0 & 23.2 \\ & & & 3.1 & & 2.1 & & & 0.8\end{array}$ $\begin{array}{llllllll}9.4 & 11.5 & 9.6 & 9.5 & 7.9 & 7.6 & 7.3 & 6.6\end{array}$ $\begin{array}{llll}6.2 & 5.8 & 5.1 & 4.9\end{array}$ $\begin{array}{llllllllllll}5.8 & 6.5 & 5.9 & 6.3 & 5.2 & 5.0 & 5.0 & 4.1 & 3.9 & 3.6 & 3.4 & 3.3\end{array}$ \begin{tabular}{ll|l|l|l|l|l|l} 
& & 0.2 & & 0.1 & 0.7
\end{tabular} $\begin{array}{rrrrrrrrrrrr}- & 6.9 & 9.5 & 9.5 & 10.8 & 11.6 & 11.7 & 11.4 & 11.2 & 11.1 & 10.8 & 11.0\end{array}$ $\begin{array}{rrrrrrrrrrrr}- & & & & 0.5 & & & 0.3 & & & 0.4 \\ 10.7 & 10.7 & 8.8 & 8.8 & 7.3 & 6.5 & 6.6 & 6.5 & 6.0 & 5.6 & 5.1 & 4.8\end{array}$ \begin{tabular}{l|l|l|l|l|l|l}
\hline & & 0.3 & 0.6 & 0.3
\end{tabular} $\begin{array}{llllllllllll}16.6 & 18.7 & 18.0 & 18.1 & 17.1 & 17.1 & 17.1 & 15.8 & 15.6 & 15.6 & 15.1 & 15.8\end{array}$ \begin{tabular}{llrlrrrr} 
& & 0.5 & & & & 0.3 \\
\hline 3.2 & 43.2 & 36.3 & 35.0 & 35.7 & 34.7 & 34.3
\end{tabular} $\begin{array}{lllllll} & 1.9 & 2.8 & 1.4\end{array}$ $\begin{array}{rrrrrrrrrrrr}- & 6.1 & 8.7 & 9.4 & 10.5 & 10.9 & 11.3 & 9.9 & 9.8 & 9.9 & 9.3 & 9.6\end{array}$ $\begin{array}{lllllll}- & 0.6 & 1.1 & 0.3\end{array}$ $\begin{array}{llllllllllll}5.2 & 5.9 & 5.6 & 5.4 & 4.9 & 4.8 & 4.6 & 4.2 & 3.9 & 3.7 & 3.4 & 3.4\end{array}$ $\begin{array}{lllllllll} & & & & 0.4 & & 0.2 & & \\ & & 0.4 & & 0.2\end{array}$ $\begin{array}{llllllllllll}4.4 & 5.5 & 7.4 & 7.5 & 7.5 & 7.7 & 8.1 & 6.5 & 6.4 & 6.3 & 6.0 & 6.1\end{array}$ $\begin{array}{llllllllllll} & & & & & 0.5 & & & 0.4 & & & \\ 3.0 & 3.2 & 2.8 & 2.8 & 2.2 & 2.1 & 2.0 & 1.8 & 1.7 & 1.6 & 1.4 & 1.3\end{array}$ \begin{tabular}{rrrrrrrrrrrrr} 
& & & & 0.3 & \multicolumn{1}{c}{0.7} & & 0.7 & & & & 0.4 \\
& 8.4 & 8.6 & 8.7 & 7.9 & 8.1 & 8.8 & 10.1 & 9.4 & 8.7 & 8.3 & 8.1
\end{tabular}

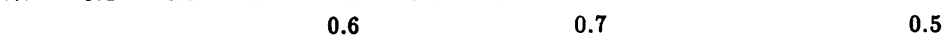
$\begin{array}{lllllllllllll}29.2^{2} & 41.0 & 47.2 & 49.9 & 49.6 & 52.2 & 52.7 & - & - & - & 43.3 & 45.6\end{array}$

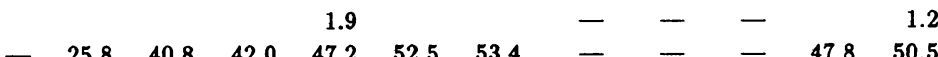
$\begin{array}{rrrrrrrrrrrrrrrrrrrrrr}\text { NGC4579 } & 4.0 & 2.4 & 2.8 & 3.3 & 2.3 & 5.6 & 10.3 & 13.6 & - & 25.8 & 40.8 & 42.0 & 47.2 & 52.5 & 53.4 & - & - & - & 47.8 & 50.5 \\ & & & 0.8 & & 0.5 & & & 0.5 & - & & & & 1.3 & & & - & - & - & & 1.0\end{array}$

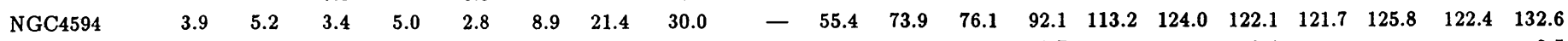

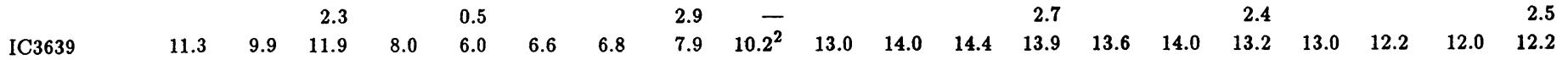
$\begin{array}{lllllll} & & & 2.7 & 0.6 & & 0.3\end{array}$ $\begin{array}{cccccccccccc}- & 5.3 & 6.6 & 6.9 & 6.9 & 7.4 & 7.4 & 7.0 & 6.8 & 6.8 & 6.4 & 6.6\end{array}$ $\begin{array}{lrrrrrrrr}\text { NGC5102 } & 26.2 & 24.6 & 24.5 & 24.2 & 18.8 & 27.1 & 35.9 & 42.9\end{array}$ $\begin{array}{rrrrrrrrr} & & & 1.0 & & 1.5 & & & 2.2 \\ \text { NGC5135 } & 10.5 & 10.6 & 9.2 & 8.9 & 10.4 & 10.9 & 10.1 & 10.3\end{array}$ $\begin{array}{rrrrrrrrrrrr}61.4 & 81.4 & 79.9 & 81.4 & 71.5 & 69.9 & 70.7 & 61.7 & 58.6 & 54.8 & 51.3 & 50.6\end{array}$

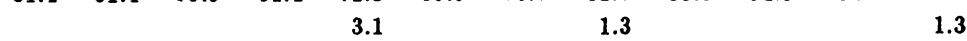
$\begin{array}{llllllllllll}13.5^{2} & 16.6 & 17.1 & 16.9 & 16.9 & 17.6 & 17.3 & 16.8 & 16.9 & 17.1 & 16.1 & 17.3\end{array}$ $\begin{array}{rrrrrrrrrrrrrrrrrrrrrr} & & & & 1.6 & & 2.3 & & 0.6 & & & & & 0.9 & & 0.6 & & & & & & \\ \text { NGC5236 } & 249.0 & 245.6 & 227.8 & 214.1 & 162.0 & 138.0 & 111.2 & 117.4 & 133.2^{2} & 152.8 & 143.4 & 146.0 & 123.1 & 118.7 & 113.3 & 103.5 & 97.1 & 94.1 & 88.7 & 93.8\end{array}$

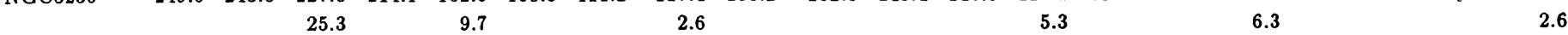

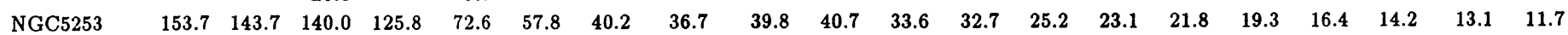

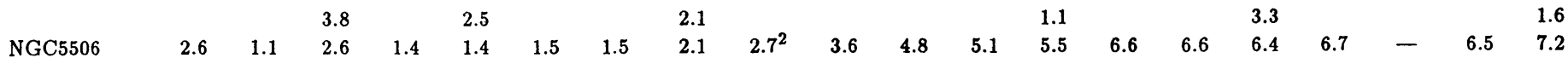
$\begin{array}{lllllllllllllllllllll}\text { NGC5506 } & 2.6 & 1.1 & 2.6 & 1.4 & 1.4 & 1.5 & 1.5 & 2.1 & 2.7^{2} & 3.6 & 4.8 & 5.1 & 5.5 & 6.6 & 6.6 & 6.4 & 6.7 & - & 6.5 & 7.2 \\ & & & 2.1 & & 0.8 & & & 0.3 & & & & & 0.4 & & & 0.7 & & - & & 0.8\end{array}$ 
TABLE 4-Continued

Wavelengths in $\AA$

\begin{tabular}{|c|c|c|c|c|c|c|c|c|c|c|c|c|c|c|c|c|c|c|c|c|}
\hline galaxy & 1355 & 1455 & 1507 & 1583 & 2530 & 2900 & 3500 & 3740 & 3810 & 4020 & 4510 & 4630 & 5313 & 5870 & 6080 & 6563 & 7043 & 7525 & 8180 & 8838 \\
\hline \multirow[t]{2}{*}{ NGC5643 } & 0.4 & 2.3 & 1.1 & 1.9 & 1.6 & 2.3 & 4.0 & 5.2 & 6.6 & 9.2 & 12.0 & 13.5 & 14.8 & 16.8 & 16.7 & 17.2 & 17.1 & - & 17.2 & 18.8 \\
\hline & & & 1.8 & & 0.5 & & & 0.5 & & & & & 0.6 & & & 1.2 & & - & & 1.0 \\
\hline \multirow[t]{2}{*}{ NGC5728 } & 3.0 & 3.2 & 3.3 & 2.6 & 2.1 & 3.4 & 4.6 & 5.5 & 7.3 & 10.1 & 12.8 & 13.7 & 14.7 & 15.6 & 15.5 & 14.6 & 12.4 & - & 15.6 & 17.1 \\
\hline & & & 1.4 & & 0.6 & & & 0.4 & & & & & 0.8 & & & 0.9 & & - & & 0.5 \\
\hline \multirow[t]{2}{*}{ NGC6221 } & 2.2: & 2.9: & $2.3:$ & 1.7: & 3.9 & 4.6 & 6.1 & 7.2 & $8.4^{2}$ & 10.9 & 13.7 & 14.3 & 14.3 & 15.8 & 16.8 & 17.9 & 17.9 & 18.1 & 18.5 & 19.6 \\
\hline & & & 2.5 & & 1.0 & & & 0.3 & & & & & 0.6 & & & 1.0 & & & & 0.8 \\
\hline \multirow[t]{2}{*}{ TOL1924_416 } & 43.4 & 39.2 & 36.2 & 31.1 & 13.4 & 11.0 & 9.7 & 9.1 & 10.4 & 10.4 & 9.6 & 9.6 & 8.4 & 7.4 & 7.3 & 6.4 & 5.4 & 5.1 & 4.2 & 3.7 \\
\hline & & & 1.2 & & 0.6 & & & 0.4 & & & & & 0.4 & & & 0.7 & & & & 0.6 \\
\hline \multirow[t]{2}{*}{1941.543} & 8.9 & 9.8 & 8.0 & 7.1 & - & - & 3.7 & 4.3 & 4.7 & 5.0 & 4.1 & 3.9 & 3.1 & 2.8 & 2.7 & 2.1 & 2.1 & 1.8 & 1.7 & 1.6 \\
\hline & & & 1.8 & & - & - & & 0.7 & & & & & 0.4 & & & 0.0 & & & & 0.3 \\
\hline \multirow[t]{2}{*}{ NGC7130 } & 7.3 & 10.5 & 7.4 & 4.9 & 7.0 & 7.6 & 8.2 & 9.6 & 12.6 & 13.5 & 13.6 & 13.3 & 12.9 & 12.9 & 12.8 & 12.4 & 12.2 & 11.9 & 10.5 & 11.1 \\
\hline & & & 1.5 & & 1.5 & & & 0.5 & & & & & 0.4 & & & 0.9 & & & & 0.4 \\
\hline \multirow[t]{2}{*}{ Mrk309 } & $1.7:$ & $2.4:$ & 2.9: & 3.3: & - & - & 2.8 & 2.8 & 2.9 & 2.9 & 2.7 & 2.6 & 2.4 & 2.3 & 2.2 & 2.1 & 1.9 & 1.9 & 1.9 & 1.9: \\
\hline & & & 1.5 & & - & - & & 0.2 & & & & & 0.2 & & & 0.3 & & & & 2.7 \\
\hline \multirow[t]{2}{*}{ NGC7496 } & 17.6 & 14.2 & 15.5 & 12.3 & 9.4 & 8.7 & 7.7 & 8.5 & 10.2 & 11.4 & 10.9 & 10.9 & 10.0 & 9.8 & 9.6 & 9.4 & 9.2 & 8.8 & 8.0 & 8.1 \\
\hline & & & 2.5 & & 0.9 & & & 0.4 & & & & & 0.4 & & & 0.6 & & & & 0.5 \\
\hline \multirow[t]{2}{*}{ NGC7552 } & 17.5 & 18.1 & 16.9 & 15.2 & 22.8 & 25.0 & 25.5 & 30.5 & 41.4 & 45.0 & 44.6 & 43.6 & 42.9 & 42.8 & 41.9 & 40.8 & 41.3 & 41.5 & 40.4 & 41.4 \\
\hline & & & 1.5 & & 1.9 & & & 0.4 & & & & & 1.4 & & & 0.5 & & & & 0.9 \\
\hline \multirow[t]{2}{*}{ NGC7582 } & 1.5 & 0.4 & 1.6 & 1.1 & 2.4 & 3.9 & 5.6 & 7.0 & 9.8 & 11.4 & 14.8 & 14.9 & 16.9 & 17.7 & 18.6 & 17.8 & 18.8 & 19.9 & 21.0 & 22.3 \\
\hline & & & 1.3 & & 0.7 & & & 0.3 & & & & & 0.9 & & & 0.4 & & & & 0.7 \\
\hline \multirow[t]{2}{*}{ NGC7590 } & 3.9 & 3.8 & 3.4 & 4.2 & 3.0 & 3.3 & 6.2 & 6.7 & $9.3^{2}$ & 12.6 & 14.3 & 15.2 & 15.9 & 17.3 & 17.5 & 17.1 & 17.1 & 17.5 & 16.9 & 17.3 \\
\hline & & & 1.4 & & 0.6 & & & 0.6 & & & & & 0.8 & & & 0.4 & & & & 0.6 \\
\hline \multirow[t]{2}{*}{ NGC7673 } & 24.0 & 24.1 & 22.0 & 18.9 & 10.3 & 9.5 & 9.6 & 10.5 & 12.1 & 16.1 & 15.2 & 15.3 & 12.1 & 11.3 & 11.0 & 10.5 & 9.7 & 8.9 & 8.8 & 8.7 \\
\hline & & & 2.0 & & 0.7 & & & 0.8 & & & & & 0.5 & & & 0.7 & & & & 1.2 \\
\hline \multirow[t]{2}{*}{ NGC7714 } & 39.9 & 38.5 & 36.5 & 33.1 & 20.0 & 19.0 & 15.0 & 15.4 & 18.4 & 19.8 & 15.8 & 16.5 & 14.6 & 13.7 & 13.7 & 12.0 & 11.5 & 10.6 & 9.7 & 9.3 \\
\hline & & & 1.3 & & 1.4 & & & 0.6 & & & & & 1.3 & & & 0.6 & & & & 0.6 \\
\hline \multirow[t]{2}{*}{ Mrk542 } & 6.6: & 8.2: & $7.2:$ & 5. $2:$ & - & - & 2.9 & 2.9 & $4.0^{2}$ & 4.2 & 4.0 & 3.9 & 3.5 & 3.3 & 3.3 & 2.6 & - & - & - & - \\
\hline & & & 1.7 & & - & - & & 0.3 & & & & & 0.2 & & & 0.5 & - & - & - & - \\
\hline \multirow[t]{2}{*}{ NGC7793 } & 12.8 & 13.5 & 13.7 & 12.2 & 5.7 & 5.9 & 6.1 & 6.2 & 9.3 & 9.9 & 10.5 & 10.5 & 9.6 & 9.4 & 9.2 & 8.5 & 8.4 & 8.3 & 7.5 & 7.2 \\
\hline & & & 2.9 & & 0.6 & & & 0.6 & & & & & 0.6 & & & 0.4 & & & & 0.3 \\
\hline
\end{tabular}

${ }^{1}$ Fluxes in units of $10^{-15} \mathrm{ergs} \mathrm{cm}^{-2} \mathrm{~s}^{-1} \AA^{-1}$. Second line shows the rms deviation from the average continuum value for selected wavelengths (see test).

${ }^{2}$ The listed flux corresponds to $\lambda 3780$ instead of $\lambda 3810$.

The above continuum points were then connected by straight lines which were used as the continua for the calculation of equivalent widths $(W)$ of the absorption features listed in Table 3. The results are presented in Table 5. A dash in the table indicates either that the corresponding segment of the spectrum is lacking or that the spectrum was too noisy in the region to give a reliable value for $W$. Typical errors in the measured $W$ are about $1 \AA$.

The fluxes of the emission lines (identified in Table 2) have then been measured for the whole sample and are listed in Tables 6 and 7: in Table 6 we present the fluxes of the main emission lines in all the spectra, and in Table 7, we list the fluxes of the emission lines in the UV, He II $\lambda 4686$, [Ar III] $\lambda 7135$, and [O II] $\lambda \lambda 7319,7330$, which can be measured only for a few galaxies. For each galaxy we present, besides the fluxes in the first line of the table, an estimate of the error in the second line. The error was calculated as the product between the rms deviation from the average continuum value adjacent to the emission line and the full width at half-maximum (FWHM) of each line, as we verified that the uncertainties in the continuum are the largest sources of errors in the measured fluxes. These errors are listed only for a few lines, as emission lines with close $\lambda$ values (e.g. $\mathrm{H} \beta$, [O III] $\lambda \lambda 4959,5007$ ) have similar FWHMs and continuum rms, and thus the same errors can be adopted.

\section{DISCUSSION}

In order to look for general trends in the observed properties, we have grouped the galaxies according to their activity type, and calculated average continuum, emission, and absorption line properties.

\subsection{Emission-Line Properties}

Table 8 shows the average emission-line ratios [O III] $\lambda \lambda 4959,5007 / \mathrm{H} \beta, \quad\left[\begin{array}{ll}\mathrm{N} & \mathrm{II}\end{array}\right] \lambda \lambda 6548,6583 / \mathrm{H} \alpha, \quad\left[\begin{array}{ll}\mathrm{S} & \mathrm{II}\end{array}\right]$ $\lambda \lambda 6717,6731 / \mathrm{H} \alpha$, [S III] $\lambda \lambda 9068,9532 / \mathrm{H} \alpha$, and $\mathrm{H} \alpha / \mathrm{H} \beta$. We also show the corresponding standard deviations for each activity type. The ratio [S III] $\lambda \lambda 9068,9532 / \mathrm{H} \alpha$ was corrected for reddening, assuming an intrinsic ratio $\mathrm{H} \alpha / \mathrm{H} \beta=2.9$. The last column lists the number of galaxies in each activity type.

The largest emission-line ratios are presented by the Sy class and are consistent with the predicted ratios for Seyferts galaxies in the diagnostic diagrams for the optical of Baldwin, Phillips, \& Terlevich ( 1981) and for the near-IR of Osterbrock, Tran, \& Veilleux (1992). The $\mathrm{H} \alpha / \mathrm{H} \beta$ ratio, although among the highest ones, is comparable to that of the SBs, composite and Hs's.

Among the non-Seyfert galaxies, there is a trend in the emission-line ratios from BCDG, through BCG and $\mathrm{H}$ II to SBs: [O III $] / \mathrm{H} \beta$ decreases while [ $\mathrm{N} \mathrm{II}] / \mathrm{H} \alpha$ increases, which is con- 
TABLE 5

EQUIVALENT WIDTHS OF ABSORPTION LINES $(\AA)$

\begin{tabular}{|c|c|c|c|c|c|c|c|c|c|c|c|c|c|c|}
\hline Galaxy & Si IV & C IV & $\mathrm{Fe}$ II & $\mathrm{Mg}$ II & $\mathrm{Ca} \mathrm{K}$ & $\mathrm{CaH}$ & $\mathrm{CN}$ & Gband & $\mathrm{Mg}$ & $\mathrm{Na} \mathrm{I}$ & TiO & CaII(1) & CaII(2) & $\mathrm{CaII}(3)$ \\
\hline Haro15 & 8.1 & 4.4 & - & - & 4.5 & 6.6 & 2.8 & 2.1 & 3.2 & 2.9 & - & - & - & - \\
\hline NGC1097 & 10.1 & 11.4 & 3.2 & 5.8 & 7.7 & 7.4 & 5.6 & 5.0 & 6.6 & 5.1 & 8.1 & 4.8 & 6.1 & 4.7 \\
\hline NGC1068 & $\mathrm{em}^{1}$ & $e m^{1}$ & 0.3 & $\mathrm{em}^{1}$ & 9.4 & 1.5 & 4.2 & 3.5 & 3.5 & 2.0 & 8.7 & 3.9 & 5.5 & 4.2 \\
\hline NGC1140 & 5.1 & 3.9 & - & - & 3.1 & 4.1 & 1.7 & 1.8 & 1.2 & 0.2 & 4.4 & 2.9 & 3.9 & 2.8 \\
\hline NGC1313 & 4.3 & 4.5 & 7.5 & 7.4 & 3.3 & 6.9 & 0.4 & 2.0 & 2.0 & -0.3 & 4.7 & 1.5 & 4.1 & 3.3 \\
\hline NGC1433 & - & - & - & - & 11.8 & 10.6 & 6.7 & 6.9 & 7.0 & 3.6 & 8.0 & 4.5 & 6.3 & 5.0 \\
\hline NGC1510 & 3.7 & 5.0 & 3.3 & 3.0 & 4.7 & 6.2 & 3.4 & 3.7 & 1.3 & 0.1 & 3.7 & - & - & - \\
\hline NGC1614 & - & 7.4 & - & - & 3.4 & 5.9 & 1.2 & 2.4 & 2.5 & 4.8 & 4.6 & 3.9 & 4.8 & 4.5 \\
\hline NGC1672 & 8.6 & 11.8 & 7.3 & 12.4 & 6.1 & 8.1 & 2.9 & 3.6 & 5.0 & 3.1 & 6.6 & 4.9 & 6.4 & 5.0 \\
\hline NGC1667 & 21.2 & 9.1 & - & - & 10.1 & 9.9 & .7 .2 & 6.9 & 6.6 & 3.8 & 7.7 & 4.0 & 5.3 & 5.3 \\
\hline NGC1705 & 5.0 & 6.3 & 4.3 & 6.5 & 2.9 & 4.5 & 1.6 & 1.2 & 3.3 & 2.7 & 5.2 & 4.0 & 4.3 & 4.7 \\
\hline NGC1800 & 6.9 & 4.3 & - & - & 3.9 & 7.9 & 2.2 & 3.1 & 1.6 & 0.8 & 5.2 & 4.8 & - & 4.8 \\
\hline NGC3049 & 7.3 & 13.3 & 5.1 & 5.5 & - & - & - & - & - & - & - & - & - & - \\
\hline NGC3081 & $\mathrm{em}^{1}$ & $\mathrm{em}^{1}$ & - & $\mathrm{em}^{1}$ & 9.9 & 6.8 & 7.2 & 7.7 & 8.2 & 4.8 & 9.7 & 4.0 & 5.6 & 4.5 \\
\hline NGC3125 & 5.9 & 3.6 & 5.0 & 5.7 & - & - & - & - & - & - & - & 3.3 & 4.6 & 3.4 \\
\hline NGC3256 & 9.4 & 10.6 & - & - & 1.7 & 2.8 & 2.3 & 0.6 & 2.9 & 4.1 & 4.0 & 3.5 & 4.8 & 3.2 \\
\hline NGC3351 & 8.6 & 17.2 & 4.8 & 7.0 & 8.6 & 8.3 & 5.0 & 3.0 & 4.4 & 3.2 & 8.1 & 4.7 & 6.3 & 5.3 \\
\hline NGC3393 & $\mathrm{em}^{1}$ & $\mathrm{em}^{1}$ & - & $\mathrm{em}^{1}$ & 15.8 & 1.5 & 10.7 & 9.4 & 8.7 & 3.8 & 10.7 & 3.3 & 4.9 & 3.7 \\
\hline $1050+04$ & 8.8 & 8.4 & 4.5 & 4.8 & 2.4 & 5.1 & 2.7 & 4.0 & 2.8 & - & - & 1.5 & 4.1 & 3.2 \\
\hline NGC3660 & - & + & 4.2 & 4.9 & 13.0 & 10.5 & 7.1 & 7.1 & 5.5 & 0.1 & 7.1 & 1.7 & 3.7 & 3.3 \\
\hline ESO572-34 & 4.7 & 6.4 & - & - & - & - & - & - & - & - & - & - & - & - \\
\hline NGC4385 & 8.3 & 11.5 & 6.6 & 8.7 & 8.1 & 5.2 & 5.0 & 2.6 & 1.5 & 2.5 & 9.7 & 3.4 & 4.5 & 3.3 \\
\hline NGC4569 & - & - & 6.1 & 8.5 & 7.5 & 10.2 & 3.2 & 3.4 & 4.5 & 3.9 & - & 2.8 & 4.8 & 4.3 \\
\hline NGC4579 & 3.0 & $\mathrm{em}^{1}$ & 1.3 & $\mathrm{em}^{1}$ & 18.0 & 12.6 & $11: 1$ & 8.6 & 7.2 & 3.8 & - & 3.3 & 5.4 & 4.2 \\
\hline NGC4594 & - & - & 3.9 & 23.0 & 17.8 & 12.2 & 11.9 & 6.9 & 7.5 & 5.1 & 11.3 & 3.6 & 5.1 & 4.3 \\
\hline IC3639 & 8.6 & 6.9 & 3.2 & 3.5 & 6.0 & 6.4 & 2.6 & 3.3 & 4.9 & 2.4 & 6.8 & 2.3 & 4.4 & 3.7 \\
\hline NGC4748 & $\mathrm{em}^{1}$ & $\mathrm{em}^{1}$ & 1.7 & $\mathrm{em}^{1}$ & 6.9 & 2.0 & 5.6 & 4.1 & 5.7 & 1.3 & 10.5 & 1.0 & 3.2 & 3.7 \\
\hline NGC5102 & 3.8 & 2.6 & 5.5 & 9.1 & 6.3 & 10.7 & 3.4 & 2.9 & 2.4 & 1.6 & 3.1 & 2.2 & 4.1 & 3.7 \\
\hline NGC5135 & 11.6 & $\mathrm{em}^{1}$ & - & - & 4.7 & 6.4 & $\begin{array}{l}. .4 \\
3.3\end{array}$ & 2.7 & 2.7 & 2.6 & $\begin{array}{l}0.1 \\
6.6\end{array}$ & 3.6 & 5.0 & 5.5 \\
\hline NGC5236 & 11.3 & 12.3 & 5.8 & 5.3 & 4.3 & 6.2 & 3.2 & 1.5 & 2.5 & 2.6 & 6.6 & 2.9 & 4.9 & 4.3 \\
\hline NGC5253 & 4.5 & 4.6 & 6.5 & 6.3 & 3.4 & $\mathrm{em}^{1}$ & 1.9 & 0.6 & 1.8 & $\mathrm{em}^{1}$ & 4.1 & - & - & - \\
\hline NGC5506 & - & - & - & - & 13.3 & 5.2 & 8.4 & 7.7 & 4.5 & 4.5 & - & 0.5 & 1.1 & 0.4 \\
\hline NGC5643 & - & $\mathrm{em}^{1}$ & - & $\mathrm{em}^{1}$ & 10.1 & 6.5 & 6.3 & 6.8 & 4.3 & 2.6 & - & 3.2 & 4.5 & 4.2 \\
\hline NGC5728 & - & $\mathrm{em}^{1}$ & - & - & 10.4 & 6.5 & 5.9 & 4.7 & 6.1 & 3.9 & - & 2.2 & 4.4 & 3.2 \\
\hline NGC6221 & - & - & - & - & 8.3 & 6.8 & 3.8 & 3.1 & 5.1 & 1.6 & 6.0 & 3.0 & 4.6 & 3.5 \\
\hline TOL1924-416 & 5.1 & 2.1 & 2.6 & $\mathrm{em}^{1}$ & 7.4 & $\mathrm{em}^{1}$ & -1.2 & 1.8 & 1.9 & $\mathrm{em}^{1}$ & 6.3 & - & - & - \\
\hline $1941-543$ & 7.5 & 1.5 & - & - & 2.5 & 6.1 & 4.8 & 3.0 & 2.1 & 1.6 & 3.8 & - & - & - \\
\hline NGC7130 & 19.8 & 21.1 & 4.7 & 0.5 & 3.8 & 5.7 & 1.2 & 2.7 & 4.7 & 4.5 & 8.8 & 3.1 & 4.8 & 5.2 \\
\hline NGC7496 & 9.4 & 11.1 & 3.5 & 6.6 & 4.5 & 6.3 & 3.5 & 3.5 & 3.2 & 3.2 & 3.5 & 3.8 & 4.8 & 4.0 \\
\hline NGC7552 & 10.2 & 11.5 & 6.9 & - & 5.2 & 8.1 & 2.4 & 2.6 & 3.2 & 5.6 & 6.1 & 4.5 & 5.5 & 4.3 \\
\hline NGC7582 & - & - & - & 9.1 & 7.6 & 7.2 & 3.2 & 3.8 & 4.8 & 4.1 & 7.8 & - & 5.9 & 4.2 \\
\hline NGC7590 & - & - & - & 10.8 & 11.7 & 9.5 & 5.6 & 7.7 & 5.0 & 4.3 & 9.2 & 4.1 & 5.9 & 5.0 \\
\hline NGC7673 & 4.7 & 7.8 & 5.3 & 6.2 & - & - & - & - & - & - & - & - & - & - \\
\hline NGC7714 & 7.5 & 8.0 & 3,5 & 6.4 & 4.1 & 2.4 & 2.0 & - & 1.6 & 0.3 & 7.5 & 4.1 & 4.6 & 2.6 \\
\hline Mrk542 & - & - & - & - & 6.0 & 7.8 & 2.4 & 2.4 & - & - & - & - & - & - \\
\hline NGC7793 & 5.6 & 9.6 & 3.3 & 6.0 & 6.7 & 10.0 & 1.6 & 3.3 & 4.0 & 1.6 & 5.6 & 4.1 & 4.6 & 3.4 \\
\hline
\end{tabular}

sistent with a decreasing gas excitation and increasing metallicity (McCall, Rybski, \& Shields 1985; Pagel \& Edmunds 1981 ). The $\mathrm{H} \alpha / \mathrm{H} \beta$ ratio also indicates an increasing reddening. The [S II $] / \mathrm{H} \alpha$ and [ $\mathrm{S} \mathrm{III]} / \mathrm{H} \alpha$ ratios do not show any clear trend, being similar in all types, except perhaps in the BCDG, which show a larger [S III]/[S II] ratio, consistent with its higher excitation. We would like to point out that the galaxy NGC 5253, although classified as SB, has all the emission line ratios, continuum and absorption line $W$ values much more similar to the BCDG type. The fact that it can be considered a dwarf galaxy $\left(M_{B}=-18.2\right)$ also favors this classification. We have thus considered NGC 5253 as a BCDG in the calculation of the average properties.

The galaxies classified as composite show [O III] $/ \mathrm{H} \beta$ and [N II] $/ \mathrm{H} \alpha$ values intermediate between SBs and Sy's (as expected), and similar $\mathrm{H} \alpha / \mathrm{H} \beta$ to both types. Nevertheless, the [S II] $/ \mathrm{H} \alpha$ and [S III] $/ \mathrm{H} \alpha$ ratios are smaller than the expected intermediate values. This is probably indicating that the starburst activity is dominating the Seyfert activity in our small sample ( 4 composite galaxies). The Hs galaxies have emissionline ratios similar to the SBs.

We also show in the seventh column of Table 8 the average $\mathrm{H} \alpha$ luminosity for each activity type, calculated using the distances listed on Table 1 and the reddening calculated as above. It can be seen that, on average, the BCDG are the less luminous and the SB are the more luminous in $\mathrm{H} \alpha$, although there is a large dispersion within each type. For this reason we also show as a second entry in the seventh column of the table, the range in $\mathrm{H} \alpha$ luminosity for each type. It can be seen that the Sy's and the composites show a similar range to that of the SB's.

Although our sample includes also three LINERs, the spectral distributions in the region $\lambda \lambda 6400-8200$ are missing (due to bad weather) for two of them. As the strongest emission lines for the LINERs are [O II] $\lambda 3727,[\mathrm{~N}$ II] $\lambda \lambda 6584,6548, \mathrm{H} \alpha$, 
TABLE 6

FLUXES OF MAIN EMISSION LiNES ${ }^{1}$

\begin{tabular}{|c|c|c|c|c|c|c|c|c|c|c|c|c|c|c|}
\hline galaxy & {$[\mathrm{OII}]_{1}$} & [NeIII] & $\mathrm{H} \gamma$ & {$[\mathrm{OIII}]_{1}$} & $\mathrm{H} \beta$ & {$[\mathrm{OIII}]_{2}$} & $\mathrm{HeI}$ & [OI] & $\mathrm{H} \alpha$ & [NII] & {$[\mathrm{SII}]_{1}$} & {$[\mathrm{SII}]_{2}$} & {$[\mathrm{SIII}]_{1}$} & {$[\mathrm{SIII}]_{2}$} \\
\hline \multirow[t]{2}{*}{ HARO15 } & 264.6 & - & 25.7 & - & 81.0 & 187.7 & - & - & 301.1 & 49.07 & 51.7 & 32.3 & - & - \\
\hline & 3.2 & - & 2.8 & - & & 2.1 & - & - & 2.5 & & & & - & - \\
\hline \multirow[t]{2}{*}{ ESO296-11 } & 135.1 & - & 18.1 & - & 36.4 & 104.5 & - & 5: & 114.9 & 21.7 & 30.6 & 18.4 & 11.6 & 41.3 \\
\hline & 3.4 & - & 1.9 & - & & 2.9 & - & & 1.2 & & & & & 3.2 \\
\hline \multirow[t]{2}{*}{ NGC1068 } & 2196.1 & 1557.6 & 352.0: & 226: & 1049.2 & 13671.3 & - & 564.7: & 7747.2 & 13910.4 & $2773.3^{2}$ & - & 2996.8 & 6618.7 \\
\hline & 16.5 & & 92.8 & & & 37.9 & - & & 176.64 & & & - & & 118.7 \\
\hline \multirow[t]{2}{*}{ NGC1097 } & 111.0 & - & 26.8: & - & 101.0 & 19.4 & - & - & 886.3 & 468.6 & 115.0 & 86.9 & - & - \\
\hline & 2.9 & - & 6.7 & - & & 5.4 & - & - & 4.7 & & & & - & - \\
\hline \multirow[t]{2}{*}{ NGC1140 } & 1010.0 & 86.0 & 121.3 & - & 350.3 & 930.5 & 47.7 & - & 1400.0 & 133.0 & 163.5 & 103.5 & 113.4 & 273.8 \\
\hline & 4.9 & & 3.1 & - & & 2.9 & & - & 3.0 & & & & & 4.4 \\
\hline \multirow[t]{2}{*}{ NGC1313 } & 105.4 & - & 3.5: & - & 17.3 & 31.0 & - & - & 148.4 & 24.8 & 33.9 & 24.0 & 13.7 & 23.0 \\
\hline & 2.6 & - & 2.5 & - & & 1.4 & - & - & 0.7 & & & & & 9.5 \\
\hline \multirow[t]{2}{*}{ NGC1433 } & 90.0 & - & - & - & - & - & - & - & 97.0 & 149.5 & 49.9 & 45.0 & - & - \\
\hline & 4.1 & - & - & - & - & - & - & - & 3.8 & & & & - & - \\
\hline NGC1510 & 340.9 & 58.2 & 31.6 & 10.1 & 116.8 & 577.8 & 24.5 & - & 512.1 & 50.7 & 49.2 & 35.3 & - & - \\
\hline & 2.1 & & 1.2 & & & 1.2 & & - & 1.4 & & & & - & - \\
\hline NGC1614 & 109.6 & - & 18.8 & - & 86.1 & 82.3 & 11.0 & 30.6 & 923.8 & 491.5 & 111.6 & 112.6 & 147.9 & 497.0 \\
\hline & 2.2 & - & 3.7 & - & & 1.8 & & & 2.2 & & & & & 6.7 \\
\hline NGC1672 & 169.2 & - & 42.0 & - & 253.1 & 98.9 & - & - & 1770.0 & 805.4 & 216.0 & 194.4 & 93.4 & 275.4 \\
\hline & 8.7 & - & 8.1 & - & & 6.2 & - & - & 5.7 & & & & & 19.3 \\
\hline NGC1667 & 43.9 & - & - & - & 18.33 & 63.8 & - & - & 178.5 & 123.3 & 38.8 & 36.2 & - & - \\
\hline & 2.0 & - & - & - & & 1.7 & - & - & 2.0 & & & & - & - \\
\hline NGC1705 & 265.0 & 52.8 & 22.3 & - & 130.0 & 599.9 & - & - & 434.1 & 16.7 & $40.0^{2}$ & - & 18.5 & 49.1 \\
\hline & 5.3 & & 5.0 & - & & 3.7 & - & - & 8.6 & & & - & & 8.1 \\
\hline NGC1800 & 199.3 & 13.0 & 4.0: & - & 26.0 & 103.8 & 15.0 & - & 125.9 & 21.7 & 29.9 & 19.0 & 15.6 & 34.4 \\
\hline & 2.5 & & 4.8 & - & & 3.2 & & - & 1.7 & & & & & 7.2 \\
\hline NGC3049 & 148.5 & - & 51.9 & - & 116.1 & 26.3 & - & - & 513.1 & 197.7 & 65.2 & 55.2 & 68.8 & 132.3 \\
\hline & 3.9 & - & 2.8 & - & & 5.7 & - & - & 1.1 & & & & & 8.6 \\
\hline NGC3081 & 69.0 & 50.7 & 12.0 & - & 44.5 & 635.9 & - & - & 195.8 & 180.5 & 42.1 & 42.0 & 83.5 & 166.2 \\
\hline & 5.0 & & 3.7 & - & & 5.1 & - & - & 2.8 & & & & & 4.5 \\
\hline NGC3125 & 744.9 & 148.9 & 149.9 & - & 368.5 & 1742.3 & 54.2 & - & 1392.0 & 84.9 & 101.6 & 76.0 & 167.6 & 326.3 \\
\hline & 3.2 & & 4.3 & - & & 5.0 & & - & 6.0 & & & & & 5.2 \\
\hline NGC3256 & 389.8 & - & 112.6 & - & 357.1 & 167.4 & 37.6 & - & 2501.0 & 1093.2 & 357.0 & 290.9 & 259.1 & 632.0 \\
\hline & 6.3 & - & 7.5 & - & & 5.5 & & - & 5.8 & & & & & 5.8 \\
\hline NGC3351 & 58.4 & - & - & - & 160.9 & - & - & - & 756.4 & 279.4 & 64.0 & 68.7 & - & - \\
\hline & 6.9 & - & - & - & 13.0 & - & - & - & 29.5 & & & & - & - \\
\hline NGC3393 & 291.2 & 119.1 & 63.0 & 23.50 & 146.0 & 1603.2 & 30.0 & - & 511.9 & 539.7 & 155.2 & 139.2 & 176.6 & 397.5 \\
\hline & 3.2 & & 6.1 & & & 4.8 & & - & 13.9 & & & & & 7.4 \\
\hline $1050+04$ & 93.3 & - & - & - & 26.0 & 43.5 & - & - & 205.2 & 68.9 & 39.2 & 26.2 & 22.2 & 44.0 \\
\hline & 3.2 & - & - & - & & 4.1 & - & - & 2.0 & & & & & 1.8 \\
\hline NGC3660 & - & - & - & - & - & 33.7 & - & - & 55.85 & 45.7 & - & - & - & - \\
\hline & - & - & - & - & - & 2.5 & - & - & 3.8 & & - & - & - & - \\
\hline ESO572-34 & 283.7 & 27.8 & 52.8 & - & 106.7 & 419.3 & - & - & 647.9 & 45.5 & 57.6 & 40.6 & 46.9 & 89.1 \\
\hline & 3.2 & & 1.7 & - & & 1.5 & 一 & - & 8.1 & & & & & 7.4 \\
\hline NGC4385 & 261.0 & - & 51.2 & - & 150.7 & 119.2 & - & - & 950.0 & 466.8 & 108.2 & 114.9 & 185.6 & 517.9 \\
\hline & 4.7 & - & 3.5 & - & & 5.6 & - & - & 5.0 & & & & & 4.0 \\
\hline NGC4569 & 109.2 & - & - & - & - & - & - & - & - & - & - & - & 23.7: & 46.4 \\
\hline & 21.1 & - & - & - & - & - & - & - & - & - & - & - & & 9.0 \\
\hline NGC4579 & 238.7 & - & - & - & - & - & - & - & - & - & - & - & - & - \\
\hline & 12.0 & - & - & - & - & - & - & - & - & - & - & - & - & - \\
\hline NGC4594 & - & - & - & - & - & - & - & - & - & 235.2 & - & - & - & - \\
\hline & - & - & - & - & - & - & - & - & - & 29.4 & - & - & - & - \\
\hline IC3639 & 209.7 & 59.1 & - & - & 85.9 & 456.5 & - & - & 477.9 & 330.1 & 116.1 & 110.5 & 110.8 & 204.3 \\
\hline & 3.5 & & - & - & & 8.5 & - & - & 5.1 & & & & & 6.9 \\
\hline NGC4748 & 61.7 & 23.1 & - & - & 57.4 & 346.8 & - & - & 334.1 & 259.8 & 44.6 & 50.9 & 51.6 & 130.2 \\
\hline & 2.8 & & - & - & & 2.7 & - & - & 8.9 & & & & & 4.4 \\
\hline NGC5102 & 95.0 & - & - & - & - & - & - & - & - & 45.6 & 37.9 & 36.8 & - & - \\
\hline & 16.6 & - & - & - & & $\overline{7}$ & - & - & 15.2 & & & & & - \\
\hline NGC5135 & 168.4 & 41.5 & - & - & 87.1 & 374.7 & - & - & 693.3 & 599.9 & 127.8 & 110.0 & 90.6 & 170.4 \\
\hline & 6.8 & & - & - & & 5.2 & - & - & 7.4 & & & & & 8.6 \\
\hline NGC5236 & 440.0 & - & 154.7 & - & 940.1 & 205.0 & - & - & 4507.0 & 2271.8 & 544.7 & 495.2 & 388.7 & 750.1 \\
\hline & 21.0 & - & 28.8 & - & & 41.6 & - & - & 66.0 & & & & & 26.5 \\
\hline NGC5253 & 4370.3 & 735.5 & 973.2 & 84.4 & 2406.0 & 11280.2 & 410.5 & 96.9 & 7717.0 & 565.8 & 643.8 & 471.2 & 970.0 & 1584.0 \\
\hline & 21.6 & & 10.7 & & & 10.6 & & & 35.0 & & & & & 14.5 \\
\hline NGC5506 & 174.9 & 37.7 & 8.3: & 2.4: & 56.9 & 521.4 & 6.8: & 60.6 & 386.6 & 354.1 & 130.8 & 118.3 & 138.2 & 315.2 \\
\hline & 2.7 & & 2.3 & & & 2.6 & & & 7.8 & & & & & 12.1 \\
\hline NGC5643 & 204.3 & 47.8 & - & - & 50.56 & 788.8 & - & 63.8: & 282.3 & 351.7 & 117.4 & 99.9 & 123.2 & 269.5 \\
\hline & 3.0 & & - & - & & 13.4 & - & & 11.9 & & & & & 13.9 \\
\hline
\end{tabular}


TABLE 6-Continued

\begin{tabular}{|c|c|c|c|c|c|c|c|c|c|c|c|c|c|c|}
\hline galaxy & {$[\mathrm{OII}]_{1}$} & [NeIII] & $\mathrm{H} \gamma$ & {$[\mathrm{OIII}]_{1}$} & $\mathrm{H} \beta$ & {$[\mathrm{OIII}]_{2}$} & $\mathrm{HeI}$ & {$[\mathrm{OI}]$} & $\mathrm{H} \alpha$ & [NII] & {$[\mathrm{SII}]_{1}$} & {$[\mathrm{SII}]_{2}$} & {$[\mathrm{SIII}]_{1}$} & {$[\mathrm{SIII}]_{2}$} \\
\hline \multirow[t]{2}{*}{ NGC5728 } & 125.3 & 56.4 & - & - & 55.5 & 677.8 & - & - & 379.1 & 403.2 & 57.3 & 56.7 & 87.7 & 223.7 \\
\hline & 4.0 & & - & - & & 8.2 & - & - & 9.9 & & & & & 7.2 \\
\hline \multirow[t]{2}{*}{ NGC6221 } & 94.4 & - & 33.9 & - & 125.2 & 71.9 & - & - & 985.1 & 642.1 & 103.5 & 113.3 & 135.0 & 346.7 \\
\hline & 3.1 & - & 5.4 & - & & 9.7 & - & - & 11.8 & & & & & 12.4 \\
\hline \multirow[t]{2}{*}{ TOL1924-416 } & 686.4 & 214.7 & 170.6 & 27.59 & 357.9 & 1744.2 & 41.8 & - & 1121.0 & 26.4 & 54.0 & 60.5 & 66.6 & 151.1 \\
\hline & $\begin{array}{r}4.4 \\
205.9\end{array}$ & 23.5 & $\begin{array}{r}3.5 \\
26.2\end{array}$ & - & 66.7 & $\begin{array}{r}5.1 \\
244.4\end{array}$ & - & $\overline{6.0}$ & $\begin{array}{r}5.7 \\
281.0\end{array}$ & 26.4 & 32.8 & 25.7 & 18.5 & $\begin{array}{r}5.3 \\
46.5\end{array}$ \\
\hline $1941-543$ & 7.1 & & 3.2 & - & . & 2.2 & - & & 0.1 & & & & & 5.7 \\
\hline \multirow[t]{2}{*}{ NGC7130 } & 129.5 & 38.7 & - & - & 70.5 & 340.9 & - & - & 560.8 & 417.9 & 76.3 & 57.2 & 38.6 & 81.7 \\
\hline & 4.1 & & - & - & & 5.2 & - & - & 11.3 & & & & & 7.0 \\
\hline \multirow[t]{2}{*}{ Mrk309 } & - & - & - & - & 19.9 & $6.0:$ & - & 一 & 111.5 & 85.4 & 7.0: & 8.0: & - & - \\
\hline & - & - & - & - & & 1.9 & - & - & 3.0 & & & & - & - \\
\hline \multirow[t]{2}{*}{ NGC7496 } & 112.9 & - & 35.4 & - & 100.9 & 66.1 & - & - & 581.9 & 279.0 & 98.5 & 67.5 & 27.1 & 62.1 \\
\hline & 3.9 & - & 5.1 & - & & 4.1 & - & - & 5.3 & & & & & 6.1 \\
\hline \multirow[t]{2}{*}{ NGC7552 } & 243.8 & - & 44.5 & - & 277.8 & 28.6 & - & 37.2 & 2064.0 & 1183.1 & 284.6 & 269.2 & 186.0 & 510.2 \\
\hline & 3.8 & - & 9.7 & - & & 9.0 & - & & 5.2 & & & & & 30.6 \\
\hline \multirow[t]{2}{*}{ NGC7582 } & 137.6 & 50.0 & 26.9 & - & 159.5 & 512.5 & 33.7: & 44.3: & 936.7 & 727.1 & 178.1 & 147.7 & 120.0 & 305.0 \\
\hline & 3.4 & & 5.4 & - & & 5.2 & & & 5.2 & & & & & 17.3 \\
\hline \multirow[t]{2}{*}{ NGC7590 } & 80.4 & - & - & - & 27.1 & 29.1 & - & - & 147.7 & 95.9 & 45.7 & 33.9 & - & - \\
\hline & 6.6 & - & - & - & & 4.7 & - & - & 3.8 & & & & - & - \\
\hline \multirow[t]{2}{*}{ NGC7673 } & 664.6 & 42.7 & 106.8 & - & 260.9 & 555.7 & 34.7 & - & 771.6 & 142.6 & 133.4 & 89.8 & 74.7 & 184.1 \\
\hline & 8.7 & & 6.4 & - & & 6.8 & & - & 6.7 & & & & & 12.7 \\
\hline \multirow[t]{2}{*}{ NGC7714 } & 860.6 & 56.9 & 171.8 & - & 423.1 & 703.1 & 90.0 & 40.9 & 1878.0 & 702.6 & 234.5 & 193.9 & 206.8 & 542.1 \\
\hline & 6.1 & & 8.0 & - & & 7.9 & & & 6.6 & & & & & 9.9 \\
\hline \multirow[t]{2}{*}{ Mrk542 } & 57.7 & - & - & - & 26.7 & 21.0 & - & 17.9 & 130.5 & 32.8 & - & - & - & - \\
\hline & 2.2 & - & - & - & & 1.4 & - & & 3.9 & & - & - & - & - \\
\hline \multirow[t]{2}{*}{ NGC7793 } & 110.6 & - & - & - & 33.0 & 18.1 & - & - & 221.9 & 74.65 & 33.8 & 25.3 & 25.3 & 76.7 \\
\hline & 7.3 & - & - & - & & 1.8 & - & - & 3.7 & & & & & 6.0 \\
\hline
\end{tabular}

${ }^{1}$ Fluxes in units of $10^{-15} \mathrm{~cm}^{-2} \mathrm{~s}^{-1}$. Second line shows error estimates for selected emission lines (see test).

${ }^{2}$ Sum of the fluxes of [S II] $\lambda \lambda 6716,6731$ emission lines.

[S II] $\lambda \lambda 6717,6731$, we are left with only the first emission line, so we have not included them in the discussion about the emission. But we would like to point out that the galaxy NGC 1433, classified as a "normal" by Kinney et al. (1993) for presenting a UV spectrum similar to a normal Sb galaxy, has all the above emission lines, with ratios typical of LINERs, confirming the classification as Seyfert-like by Veron-Cetty \& Véron 1986). Although Sersic \& Pastoriza (1965) point out the presence of an amorphous nucleus, and Buta (1986) of a nuclear ring or lens of about $20^{\prime \prime}$ diameter, the ring is barely outside our aperture. This galaxy thus seems to be another example of a Hot Spot nucleus with a LINER inside, like NGC 1097, but for which, due to its proximity, we have observed only the LINER nucleus.

\subsection{Continuum Properties}

We show in Table 9 two continuum colors, $C(14-35)$ and $C(40-53)$, defined as

$$
\begin{aligned}
& C(14-35)=-2.5 \log \frac{F_{\lambda 1355}}{F_{\lambda 3500}} \\
& C(40-53)=-2.5 \log \frac{F_{\lambda 4020}}{F_{\lambda 5313}} .
\end{aligned}
$$

There is a clear sequence in the colors, with the BCDGs being the bluest galaxies, followed by the BCGs, then the $\mathrm{H}$ II's, the SBs and the Sy. The composite type presents a redder $C(14-35)$ color than the Sy (although the standard deviation is very high), indicating that some host galaxies are particularly red (or reddened). The LINERs have the reddest colors, and the Hs show similar values to those of the Sy. These results are in agreement with the average UV slopes found by Kinney et al. (1993). We also show in the last line of the table, the continuum colors of NGC 1433: its $C(40-54)$ color is similar to those of the LINERs, although its $C(14-35)$ color is somewhat bluer.

\subsection{Absorption Lines}

We have calculated the average $W$ values of the absorption features which we concluded were present and best defined in most of the spectra. The results are presented in Table 10. The average $W$ of the UV absorption lines of Si IV and C IV increase from about $5 \AA$ in the BCDG to about $9 \AA$ in the SBs. In the Sy's, these lines are most frequently found in emission, but they reach about $14 \AA$ in the composites and Hs's. The behavior of $\mathrm{Fe}$ II and $\mathrm{Mg}$ II is less clear: there seems to be a mild gradient in $\mathrm{Mg}$ II, which increases from 5.6 $\AA$ in the BCDGs to $9.4 \AA$ in the composites, but it should be considered with caution as both $\mathrm{Mg}$ II and Fe II can have interstellar contributions from the galaxy itself and also from the Milky Way (Kinney et al. 1993).

In the optical, the $W(\mathrm{Ca}$ II $\mathrm{K})$ is about the same $(\approx 4 \AA)$ from the BCDG's through the SBs, reaching the largest value $\left(18{ }^{\circ} \mathrm{A}\right)$ for one LINER. This is about the largest value also found in normal galaxies (Bica 1988). In the Sy's, Hs's and composites, $W(\mathrm{Ca}$ II $\mathrm{K})$ has an intermediate value, which could be due to a combination of a old red population plus 
TABLE 7

FLUXES OF LESS COMMON EMISSION LINES ${ }^{\prime}$

\begin{tabular}{|c|c|c|c|c|c|c|c|c|c|c|c|}
\hline galaxy & Ly $\alpha$ & CIV & $\mathrm{HeII}_{1}$ & CIII] & $\mathrm{CII}]$ & [NeIV] & MgII & {$[\mathrm{NeV}]$} & $\mathrm{HeII}_{2}$ & [ArIII] & {$[\mathrm{OII}]_{2}$} \\
\hline \multirow[t]{2}{*}{ NGC1068 } & - & 3876.1 & 1319.7 & 1839.5 & 476.2 & 804.4 & 588.3 & 2037.6 & 388.2 & - & - \\
\hline & - & 25.0 & & 27.2 & & & 62.0 & 71.2 & 37.9 & - & - \\
\hline \multirow[t]{2}{*}{ NGC1140 } & - & - & - & - & - & - & - & - & - & 30.0 & - \\
\hline & - & - & - & - & - & - & - & - & - & 3.0 & - \\
\hline \multirow[t]{2}{*}{ NGC1510 } & - & - & - & - & - & - & 47.5: & - & - & 15.6 & 9.2 \\
\hline & - & - & - & - & - & - & 39.4 & - & - & 1.4 & \\
\hline \multirow{2}{*}{ NGC1614 } & - & - & 19.5: & - & - & - & - & - & - & 13.5 & 12.2 \\
\hline & - & - & 2.4 & - & - & - & - & - & - & 2.2 & \\
\hline \multirow[t]{2}{*}{ NGC3081 } & - & 199.3 & 75.5 & 55.2 & - & 40.5 & - & 44.2 & 20.9 & 10.7 & - \\
\hline & - & 9.7 & & 3.0 & - & 7.7 & - & 7.1 & 5.1 & 2.8 & - \\
\hline \multirow[t]{2}{*}{ NGC3125 } & - & - & - & - & - & - & - & - & - & 43.1 & 21.0 \\
\hline & - & - & - & - & - & - & - & - & - & 5.8 & \\
\hline \multirow[t]{2}{*}{ NGC3393 } & 964.1 & 312.5 & 190.0 & 98.7 & - & 52.8 & 72.9 & 58.2 & 38.2 & 39.8 & - \\
\hline & & 6.7 & & 2.7 & - & & 11.5 & 6.3 & 4.8 & 13.9 & - \\
\hline \multirow{2}{*}{ ESO572-34 } & - & - & - & 44.9 & - & - & - & - & - & - & - \\
\hline & - & - & - & 5.6 & - & - & - & - & - & - & - \\
\hline \multirow[t]{2}{*}{ NGC4385 } & - & - & - & - & 36.3 & - & - & - & - & - & - \\
\hline & - & - & - & - & 10.8 & - & - & - & - & - & - \\
\hline \multirow{2}{*}{ NGC4579 } & - & 77.7 & - & 53.6 & - & - & 24.8 & - & - & - & - \\
\hline & - & 6.9 & - & 4.7 & - & - & 1.1 & - & - & - & - \\
\hline \multirow[t]{2}{*}{ IC3639 } & - & - & - & - & - & - & - & 39.4 & - & - & 23.0 \\
\hline & - & - & - & - & - & - & - & 9.7 & - & - & 5.1 \\
\hline \multirow[t]{2}{*}{ NGC4748 } & 431.2 & 242.8 & 88.7 & 112.8: & - & - & 104: & 18.8: & - & - & - \\
\hline & & 39.9 & & 29.6 & - & - & 9.8 & 3.3 & - & - & - \\
\hline \multirow[t]{2}{*}{ NGC5135 } & 509.6 & 80.8 & 85.7 & - & - & - & - & 24.8 & - & - & - \\
\hline & & 11.5 & & - & - & - & - & 7.7 & - & - & - \\
\hline \multirow[t]{2}{*}{ NGC5253 } & - & - & - & 398.9 & - & - & - & - & - & 263.0 & 122.2 \\
\hline & - & - & - & 32.7 & - & - & - & - & - & 35.0 & \\
\hline \multirow[t]{2}{*}{ NGC5506 } & - & - & - & 59.3: & - & - & - & 6.4 & 7.4: & - & - \\
\hline & - & - & - & 11.6 & - & - & - & 2.4 & 2.6 & - & - \\
\hline \multirow[t]{2}{*}{ NGC5643 } & - & 85.7 & - & 42.1 & - & - & 22.7: & 62.4 & 20.1 & - & - \\
\hline & - & 13.2 & - & 7.3 & - & - & 4.8 & 11.7 & 13.4 & - & - \\
\hline \multirow[t]{2}{*}{ NGC5728 } & - & 89.6 & - & - & - & - & - & - & - & - & - \\
\hline & - & 12.1 & - & - & - & - & - & - & - & - & - \\
\hline \multirow[t]{2}{*}{ TOL1924-416 } & 1131.0 & - & - & 85.7 & - & - & 73.0 & - & - & 28.5 & - \\
\hline & & - & - & 2.4 & - & - & 5.9 & - & - & 5.7 & - \\
\hline \multirow[t]{2}{*}{$1941-543$} & 73.8 & - & - & - & - & - & - & - & - & - & - \\
\hline & & - & - & - & - & - & - & - & - & - & - \\
\hline \multirow[t]{2}{*}{ NGC7130 } & 202.0 & - & - & - & - & - & - & - & - & - & - \\
\hline & & - & - & - & - & - & - & - & - & - & - \\
\hline \multirow[t]{2}{*}{ NGC7582 } & - & - & - & - & - & - & - & - & 13.4: & - & - \\
\hline & - & - & - & - & - & - & - & - & 5.2 & - & - \\
\hline NGC7714 & - & - & - & - & - & - & - & - & - & 32.5 & - \\
\hline & - & - & - & - & - & - & - & - & - & 6.6 & - \\
\hline
\end{tabular}

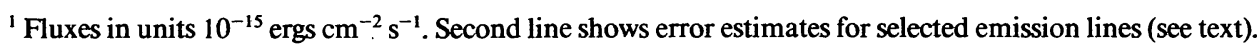

TABLE 8

AVERAGE EMISSION-LINE RATIOS PER ACTIVITY TYPE

\begin{tabular}{|c|c|c|c|c|c|c|c|}
\hline Activity Type & $N$ & {$[\mathrm{O} \mathrm{III}] / \mathrm{H} \beta$} & {$[\mathrm{N} \mathrm{II}] / \mathrm{H} \alpha$} & {$[\mathrm{S} \mathrm{II}] / \mathrm{H} \alpha$} & {$[\mathrm{S} \mathrm{III}] / \mathrm{H} \alpha$} & $\mathrm{H} \alpha / \mathrm{H} \beta$ & $L(\mathrm{H} \alpha)^{\mathbf{a}}$ \\
\hline 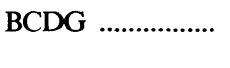 & 4 & $6.7 \pm 0.6$ & $0.09 \pm 0.03$ & $0.13 \pm 0.03$ & $0.23 \pm 0.09$ & $3.9 \pm 0.4$ & $\begin{array}{l}5.3 \pm 3.2 \\
1.0-9.9\end{array}$ \\
\hline BCG & 4 & $3.8 \pm 1.6$ & $0.20 \pm 0.15$ & $0.22 \pm 0.08$ & $0.17 \pm 0.03$ & $4.7 \pm 1.9$ & $\begin{array}{c}142.3 \pm 110.0 \\
64.4-332.2\end{array}$ \\
\hline 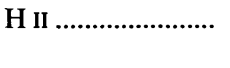 & 6 & $3.6 \pm 1.7$ & $0.22 \pm 0.11$ & $0.28 \pm 0.09$ & $0.19 \pm 0.08$ & $5.6 \pm 1.8$ & $\begin{array}{r}28.4 \pm 38.1 \\
0.4-105.9\end{array}$ \\
\hline SB & 8 & $0.8 \pm 0.7$ & $0.66 \pm 0.15$ & $0.23 \pm 0.04$ & $0.22 \pm 0.08$ & $6.2 \pm 2.0$ & $\begin{array}{r}631 \pm 789.4 \\
11.36-2264.0\end{array}$ \\
\hline 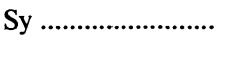 & 12 & $11.5 \pm 6.2$ & $1.24 \pm 0.40$ & $0.47 \pm 0.14$ & $0.53 \pm 0.25$ & $6.3 \pm 1.6$ & $\begin{array}{l}256 \pm 344.2 \\
10.8-1155.0\end{array}$ \\
\hline Composite ............ & 5 & $2.6 \pm 2.4$ & $0.81 \pm 0.17$ & $0.27 \pm 0.05$ & $0.13 \pm 0.06$ & $6.9 \pm 0.9$ & $\begin{array}{c}228.6 \pm 243.2 \\
36.0-706.9\end{array}$ \\
\hline 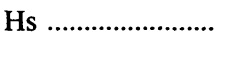 & 2 & $0.26^{\mathrm{b}}$ & $0.59 \pm 0.10$ & $0.21 \pm 0.03$ & $\cdots$ & $6.7 \pm 2.0$ & $\begin{array}{r}59.7 \pm 52.1 \\
7.6-111.7\end{array}$ \\
\hline
\end{tabular}

${ }^{\mathrm{a}}$ In units of $10^{40} \mathrm{ergs} \mathrm{s}^{-1}$.

${ }^{b}$ Only one measurement. 
TABLE 9

AVERAGe CONTINUUm COLORS PER ACTIVITY TYPE

\begin{tabular}{|c|c|c|}
\hline Activity Type & $C(14-35)$ & $C(40-53)$ \\
\hline BCDG ................. & $-1.59 \pm 0.47$ & $-0.51 \pm 0.05$ \\
\hline 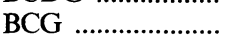 & $-1.34 \pm 0.28$ & $-0.35 \pm 0.14$ \\
\hline H II ..................... & $-1.14 \pm 0.63$ & $-0.36 \pm 0.16$ \\
\hline SB & $-0.31 \pm 0.66$ & $-0.15 \pm 0.10$ \\
\hline 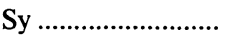 & $0.06 \pm 0.47$ & $0.34 \pm 0.16$ \\
\hline LINER ................. & $1.43 \pm 0.41^{\mathrm{a}}$ & $0.48 \pm 0.19^{b}$ \\
\hline Composite ............ & $0.36 \pm 0.82$ & $0.14 \pm 0.20$ \\
\hline 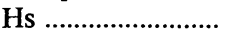 & $-0.09 \pm 0.12$ & $0.27 \pm 0.12$ \\
\hline N1433 .................... & 0.32 & 0.43 \\
\hline
\end{tabular}

a Average of only two measurements.

${ }^{b}$ Average of only three measurements.

young blue stars in Hs's and composites or a blue featureless continuum in the Sy's (Kinney et al. 1991; Schmitt, StorchiBergmann, \& Baldwin 1994). The other optical absorption features: $G$ band, $\mathrm{Mg}$ I and $\mathrm{Na}$ I, which originate mainly in the red star population, have very small $W$ in the BCDGs, BCGs, $\mathrm{H}$ II's, and SBs, indicating the dominance of blue stars in the integrated continuum in these types. For the Sy's, the average values are as large as the one for the LINERs, indicating that the dominant stellar population is old (Storchi-Bergmann, Bica, \& Pastoriza 1990) and that the dilution by a blue featureless continuum is smaller (if any) at wavelengths larger that $\approx 4000 \AA$

In the last column of Table 10 , we show $W(\mathrm{Ca}$ II $\lambda 8662)$. We have observed that the $\mathrm{Ca}$ II triplet in the BCDGs, BCGs, and $\mathrm{H}$ II's was either present with average $W(\mathrm{Ca}$ II $\lambda 8662) \approx$ 3-4 $\AA$ or not present at all. In the SBs the lines are always present, with average $W$ about the same. In stars, the $W$ of Ca II triplet correlates strongly with surface gravity, being much larger in giants and supergiants than in dwarfs (Jones, Alloin, \& Jones 1984). The Ca II triplet in the spectra is thus indicating the presence of giants and supergiants, which are always present in the SBs but not always is the BCDGs, BCGs and H II's, probably due to their absence at early ages of the burst. For the Sy's and composites, $W(\mathrm{Ca}$ II $)$ is somewhat larger, and the largest values $(5.0 \AA)$ are the ones from the Hs's and NGC 1433. Bica \& Alloin (1987) have shown that $W$ (Ca II) is sensitive to metallicity if a whole range $-2.0<Z / Z_{\odot}<0.0$ is considered. Nevertheless, there is a lot of scatter and a smaller correlation if only the range $-1.0<Z / Z_{\odot}<0.0$ is considered, which is our case (Storchi-Bergmann et al. 1994).

\subsection{Discussion of Individual Spectra}

A common characteristic to all the spectra with active star formation (BCG, BCDG, $\mathrm{H}$ II, starbursts, composite, and hot spot galaxies) is the presence in the UV of the absorption features of young blue O, B stars (e.g., Si IV $\lambda 1400$, C IV $\lambda 1550$ ) from the present burst of star formation. In the optical range, there is a varying strength of the absorption features, which denote the relative importance of previous bursts of star formation. A general trend (with a few exceptions) is: the higher the abundance (as can be hinted from the $[\mathrm{N}$ II $] / \mathrm{H} \alpha$ ratio), more conspicuous are the absorption features from previous generations of stars. The emission-line spectra vary from high excitation (high [O III] $/ \mathrm{H} \beta$ ratio) for the lower abundance galaxies (BCG, BCDG) to a decreasing excitation as the abundances get higher for the starbursts and hot spot galaxies. We now briefly discuss the different kinds of spectra we obtained for each activity class.

Figure 3 shows the spectral distributions for three BCDGs. NGC 5253 presents very similar spectrum to NGC 3125 , confirming our classification of this galaxy as a BCDG. Notice the high [O III ] $/ \mathrm{H} \beta$ and small [N $\mathrm{II}] / \mathrm{H} \alpha$ ratio and the almost absence of absorption lines in the optical spectrum. NGC 1705 differs from the two previous ones in presenting relatively strong absorption features in the optical and near-IR due to the presence of an older burst of star formation (Meurer et al. 1992). It is one of the bluest galaxies of the sample.

Figure 4 shows three examples of spectra of BCD galaxies. Tol1 924-416 shows a very similar spectrum to the previous BCDG, the only difference being that it is a more luminous galaxy. NGC 1140 and $1050+04$ show a lower excitation and higher abundance as indicated by the higher $[\mathrm{N} \mathrm{II}] / \mathrm{H} \alpha$.

Two spectra of $\mathrm{H}$ II galaxies are shown in Figure 5: NGC 7793 presents a low excitation spectrum with higher abun-

TABLE 10

AVERAGE EQUIVALENT WIDTHS PER ACTIVITY TYPE

\begin{tabular}{|c|c|c|c|c|c|c|c|c|c|}
\hline Activity Type & Si IV & CiV & Fe II & Mg II & $\mathrm{Ca} \mathrm{K}$ & $G$ Band & $\mathrm{Mg}$ & $\mathrm{Na} I$ & Ca II (3) \\
\hline BCDG & $4.8 \pm 0.8$ & $4.8 \pm 0.9$ & $5.1 \pm 1.2$ & $5.6 \pm 1.6$ & $3.7 \pm 08$ & $1.8 \pm 1.3$ & $2.1 \pm 0.8$ & $1.4 \pm 1.3^{b}$ & $4.1 \pm 0.7^{b}$ \\
\hline 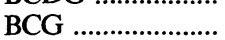 & $6.8 \pm 1.7$ & $4.7 \pm 2.3$ & $3.6 \pm 1.0^{\mathrm{b}}$ & $4.8^{\mathrm{a}}$ & $4.4 \pm 1.9$ & $2.4 \pm 0.9$ & $2.3 \pm 0.8$ & $1.6 \pm 1.4^{b}$ & $3.0 \pm 0.2^{\mathrm{b}}$ \\
\hline 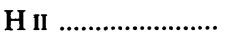 & $5.6 \pm 1.2$ & $5.7 \pm 2.6$ & $5.4 \pm 1.7^{c}$ & $6.5 \pm 0.6^{c}$ & $4.1 \pm 1.6$ & $2.9 \pm 0.5$ & $2.4 \pm 0.9$ & $0.9 \pm 0.8$ & $3.8 \pm 0.7^{\mathrm{c}}$ \\
\hline $\mathrm{Sb}$ & $8.8 \pm 2.5$ & $9.7 \pm 3.3$ & $5.6 \pm 1.1$ & $7.0 \pm 1.6$ & $4.7 \pm 1.9$ & $2.1 \pm 0.8$ & $2.4 \pm 0.6$ & $3.1 \pm 1.7$ & $3.7 \pm 0.7$ \\
\hline Sy & $\mathrm{em}^{\mathrm{d}}$ & $e^{d}$ & $2.4 \pm 1.5$ & $\mathrm{em}^{\mathrm{d}}$ & $10.1 \pm 3.0$ & $6.0 \pm 2.1$ & $5.5 \pm 1.7$ & $3.0 \pm 1.4$ & $4.2 \pm 0.8$ \\
\hline LINER $^{\mathrm{c}}$ & $\ldots$ & ... & $3.8 \pm 2.0$ & $\ldots$ & $14.4 \pm 4.9$ & $6.3 \pm 2.2$ & $6.4 \pm 1.3$ & $4.3 \pm 0.6$ & $4.3 \pm 0.1$ \\
\hline Composite ................ & $12.6 \pm 5.1^{\mathrm{c}}$ & $14.7 \pm 4.6^{c}$ & $5.2 \pm 1.6^{\mathrm{c}}$ & $9.4 \pm 2.4^{c}$ & $6.3 \pm 1.5$ & $3.3 \pm 0.4$ & $4.6 \pm 0.7$ & $3.3 \pm 1.0$ & $4.4 \pm 0.6$ \\
\hline $\mathrm{Hs}^{\mathrm{b}}$ & $9.4 \pm 0.8$ & $14.3 \pm 2.9$ & $4.0 \pm 0.8$ & $6.4 \pm 0.6$ & $8.2 \pm 0.5$ & $4.0 \pm 1.0$ & $5.5 \pm 1.1$ & $4.2 \pm 1.0$ & $5.0 \pm 0.3$ \\
\hline N1433 ..................... & $\ldots$ & $\ldots$ & $\ldots$ & $\ldots$ & 11.8 & 6.9 & 7.0 & 3.6 & 5.0 \\
\hline
\end{tabular}

a Only one measurement.

b Average of only two measurements.

${ }^{c}$ Average of only three measurements.

d In emission. 
dance than the previous types as indicated by $[\mathrm{N} \mathrm{II}] / \mathrm{H} \alpha$. Several absorption lines in the optical and the $\mathrm{Ca}$ II triplet in the near-IR can easily be seen. NGC 7673 shows somewhat larger excitation and no absorption features in the optical and near-IR.

We show in Figure 6 three examples of starbursts: NGC 7714 is the prototype starburst galaxy (Weedman et al. 1981): it is very blue, occurs in a Sdm galaxy, shows high excitation and weak absorption features in the optical and near-IR. NGC 7552 is a red starburst occurring in a SBbc galaxy, showing strong absorption lines in the optical and near-IR, indicating a mixture of moderately young and old red stellar population. The emission spectrum indicates low excitation and high chemical abundance. NGC 5236 presents very similar $W$ of absorption lines and emission-line ratios to NGC 7552, although its continuum is bluer. The difference between the two spectra seems to be due only to reddening effects produced by the different orientation of the galaxies relative to the line of sight (Kinney et al. 1994).

We show in Figure 7 two spectra of composite-type galaxies: the high [O III] $/ \mathrm{H} \beta$ and [ $\mathrm{N} \mathrm{II]} / \mathrm{H} \alpha$ of NGC 7582 indicates that its emission is dominated by the Seyfert nucleus, while in NGC 1672 the lower ratios indicate that the starburst dominates.

The spectra of the hot spot galaxies are shown in Figure 8: the emission-line ratios are similar to those of the red starbursts, only the equivalent width of the emission lines is smaller, due to a smaller starburst (concentrated in the hot spots). The optical absorption lines indicate that the red underlying population dominates, while in the starbursts there is strong contribution from moderately young stars as shown by the high-order Balmer absorption lines in the spectral region $\lambda \lambda 3700-4000$.

Figure 9 shows the spectra of two Seyfert 2 galaxies: notice the high $[\mathrm{O} \mathrm{III}] / \mathrm{H} \beta,[\mathrm{N} \mathrm{II}] / \mathrm{H} \alpha$ and $[\mathrm{S} \mathrm{III}] / \mathrm{H} \alpha$ ratios as compared to the previous galaxies. NGC 3081 occurs in a galaxy presenting a red stellar population while IC 3639 shows contribution of blue stars in the absorption spectrum.

Finally, in Figure 10, we present the spectra of the LINERs NGC 4579 and NGC 1433. The two spectra are similar in presenting red stellar populations and a "rising branch" in the UV, as well as low excitation emission lines with relatively small equivalent widths, confirming the classification of NGC 1433 as a LINER.

\section{SUMMARY AND CONCLUSIONS}

We have presented the spectral energy distributions from the ultraviolet to the near-infrared as well as the measurements of a number of spectral features for a sample of 48 galaxies classified as BCG, H II, starburst, Seyfert 2, and composite (Seyfert + starburst). The spectra were obtained through a matched large $\left(10^{\prime \prime} \times 20^{\prime \prime}\right)$ aperture. A preliminary analysis of the data was made by grouping the galaxies according to their activity type and calculating average properties. In this section, we summarize the main results.

The Seyfert galaxies present the largest $[\mathrm{O} \mathrm{III}] / \mathrm{H} \beta$ and [N II] $/ \mathrm{H} \alpha$ ratios. There is a trend in these ratios from $\mathrm{BCDG}$, through $\mathrm{BCG}$ and $\mathrm{H}$ II to SBs: [O III] $/ \mathrm{H} \beta$ decreases while [ $\mathrm{N} \mathrm{II}] / \mathrm{H} \alpha$ increases, which is consistent with a decreasing gas excitation and increasing metallicity. The $\mathrm{H} \alpha / \mathrm{H} \beta$ ratio also indicates an increasing reddening along this sequence. The Seyfert galaxies present $\mathrm{H} \alpha / \mathrm{H} \beta$ ratios among the highests, although comparable to that of the starbursts, suggesting that we are reaching similar optical depths. We are thus not probing (through the Balmer ratio) the large obscuration frequently found in the Seyfert 2 galaxies through other methods (e.g., Storchi-Bergmann, Wilson, \& Baldwin 1992; Schmitt et al. 1994). In our sample, the Seyfert and the starburst galaxies have similar $\mathrm{H} \alpha$ luminosities.

The continuum is bluest for the BCGDs, followed by the BCGs, then the H II's, the SBs, and Sy's. The three LINERs of the sample present the reddest colors.

The equivalent widths of the measured absorption lines in the ultraviolet ( $\mathrm{Si}$ IV, C IV, Fe II, and $\mathrm{Mg}$ II) increase along the sequence from BCDG to starbursts, while in the Seyfert galaxies, these absorption lines are absent, being frequently replaced by emission. In the optical, from the BCDG through the starbursts, the equivalent widths of the measured absorption features (e.g., $G$ band, $\mathrm{Mg}$ I and $\mathrm{Na}$ I, which originate mainly in the red stars) present small values, indicating the dominance of blue stars in the integrated continuum in these types. The larger values are found for the Seyfert galaxies (except for a few cases) and LINERs, indicating that the old stellar population dominates in these galaxies due to our large aperture.

A more detailed analysis of the data, incorporating both the northern and southern samples is presented in Storchi-Bergmann et al. (1994), Calzetti et al. (1994), and Kinney et al. (1994).

We would like to thank the staff at CTIO for assistance with the observations and reductions, in particular Steve Heathcote, for suggestions about the setups for the observations, and $\mathbf{M}$. Navarrete, for valuable help with the reductions. We are indebted to $\mathrm{C}$. Winge for kindly obtaining some optical spectra for us, and to K. McQuade for preparing the plots. We also benefited from valuable discussions with $\mathrm{D}$. Calzetti, T. Heckman, C. Robert, E. Bica, and C. Bonatto. T.S.B. thanks the hospitality of STScI, where part of this work was done, and the Brazilian institutions CNPq, CAPES, and FAPERGS for partial support. The authors also acknowledge support from the NASA grants NAG5-2498 and NAGW-3757.

\section{REFERENCES}

Baldwin, J. A., Phillips, M. M., \& Terlevich, R. 1981, PASP, 93, 5

Bica, E. 1988, A\&A, 195, 76

Bica, E., \& Alloin, D. 1987, A\&A, 186, 49

Buta, R. 1986, ApJS 61, 631
Calzetti, D., Kinney, A. L., \& Storchi-Bergmann, T. 1994, ApJ, 429, 582

Jones, J. E., Alloin, D., \& Jones, B. J. T. 1984, ApJ, 283, 457

Kinney, A. L., Antonucci, R. R. J., Ward, M. J., Wilson, A. S., \& Whittle, M. 1991, ApJ, 377, 100 
Kinney, A. L., Bohlin, R. C., Calzetti, D., Panagia, N., \& Wyse, R. F. G. 1993, ApJS, 86, 5

Kinney, A. L., Calzetti, D., Bica, E., \& Storchi-Bergmann, T. 1994, ApJ, 429,172

Kinney, A. L., Calzetti, D., Bohlin, R. C., McQuade, K., \& Storchi-Bergmann, T. 1995, ApJ, submitted

ItI McCall, M. L., Rybski, P. M., \& Shields, G. A. 1985, ApJS, 57, 1

McQuade, K., Calzetti, D., \& Kinney, A. L. 1995, ApJ, in press

Meurer, G. R., Freeman, K. C., Dopita, M. A., \& Cacciari, C. 1992, AJ, 103,60

Osterbrock, D. E., Tran, H. D., \& Veilleux, S. 1992, ApJ, 398, 196
Pagel, B. E., \& Edmunds, M. G. 1981, ARA\&A, 19, 77

Robert, C., Leitherer, C., \& Heckman, T. M. 1993, preprint

Schmitt, H. R., Storchi-Bergmann, T., \& Baldwin, J. 1994, ApJ, 423, 237

Sersic, J. L., \& Pastoriza, M. G. 1965, PASP, 77, 287

Storchi-Bergmann, T., Bica, E., \& Pastoriza, M. G. 1990, MNRAS, 245, 749

Storchi-Bergmann, T., Calzetti, D., \& Kinney, A. L. 1994, ApJ, 429, 572

Storchi-Bergmann, T., Wilson, A. S., \& Baldwin, J. A. 1992, ApJ, 396, 45

Véron-Cetty, M.-P., \& Véron, P. 1986, A\&AS 66, 335

Weedman, D. W., Feldman, F. R., Balzano, V. A., \& Ramsey, L. W. 1981, ApJ, 248, 105 